\title{
Partiality and its dual in natural implicative expansions of Kleene's strong 3-valued matrix with only one designated value
}

\author{
Gemma Robles* and José M. Méndez** \\ *Dpto. de Psicología, Sociología y Filosofía, Universidad de León \\ Campus de Vegazana, s/n, 24071, León, Spain \\ gemma.robles@unileon.es; http://grobv.unileon.es \\ **Universidad de Salamanca. \\ Edificio FES, Campus Unamuno, 37007, Salamanca, Spain. \\ sefus@usal.es; http://sites.google.com/site/sefusmendez
}

\begin{abstract}
Equivalent overdetermined and underdetermined bivalent Belnap-Dunn type semantics for the logics determined by all natural implicative expansions of Kleene's strong 3-valued matrix with only one designated value are provided.

Keywords: Kleene's strong 3-valued matrix; natural conditionals; BelnapDunn type bivalent semantics; 3 -valued logics; paraconsistent logics.
\end{abstract}

\section{Introduction}

The aim of this paper is to provide equivalent overdetermined and underdetermined bivalent Belnap-Dunn type semantics for the logics determined by all natural implicative expansions of Kleene's strong 3-valued matrix with only one designated value. Let us explain with detail this aim. We begin by briefly discussing the notions "Belnap-Dunn semantics", "natural conditionals" and "Kleene's strong 3-valued matrix" (we refer to some of the preliminary definitions in $\S 2$ or in other sections below).

Belnap-Dunn semantics (BD-semantics) originates with Belnap and Dunn's well-known logic B4 introduced to treat inconsistent and incomplete situations (cf. [5], [6], [7] and [8]). BD-semantics is characterized by the possibility of assigning $\mathrm{T}$ (truth), $\mathrm{F}$ (falsity), both $\mathrm{T}$ and $\mathrm{F}$ or neither $\mathrm{T}$ nor $\mathrm{F}$ to the formulas of a given formal language, or, indeed, to the propositions of a language in general. Conjunction, disjunction and negation are the only connectives in B4, but Belnap and Dunn's approach has been generalized to the notion of a bilattice, which was found important applications in artificial intelligence (cf., e.g., [2] and [3]). There are two special types of BD-semantics, underdetermined 
BD-semantics (u-semantics) and overdetermined BD-semantics (o-semantics). Formulas can be assigned T, F or neither $\mathrm{T}$ nor $\mathrm{F}$ in the former; $\mathrm{T}, \mathrm{F}$ or both $\mathrm{T}$ and $\mathrm{F}$, in the latter. U-semantics is a "partial" semantics and o-semantics is its dual. We refer the reader to Dunn's classical paper [8] for more information on the topic.

On the other hand, given a matrix semantics, a conditional is "natural" if it fulfills the following three conditions: (1) it coincides with the classical conditional when restricted to the classical values $\mathrm{T}$ and $\mathrm{F}$; (2) it satisfies the Modus Ponens; and (3) it is assigned a designated value whenever the value assigned to its antecedent is less than or equal to the value assigned to its consequent. The notion of a "natural conditional" is understood as defined in [24] (cf. Definition 2.7), but it has to be remarked that stricter notions can be found in the literature (cf., e.g., [4]).

In the third place, Kleene's strong 3-valued matrix MK3 (our label) was defined in [10] in the context of the treatment of partial recursive functions. The matrix MK3 can be defined as shown in Definition 2.6. We can take either 2 as the only designated value or else both 1 and 2 . In the former case 1 can be interpreted as neither truth nor falsity; in the latter, as both truth and falsity. The value 2 is, of course, truth, while 0 is falsity.

There are exactly 24 natural implicative expansions of MK3 with 1 and 2 as designated values, and 6 with 2 as the only designated value. By using a BD-semantics we have axiomatized in a general and unified way the logics determined (cf. Definition 7.4) by these 30 natural implicative expansions of MK3. As it was to be expected, an o-semantics is used for the logics determined by the 24 expansions of MK3 with two designated values (cf. [17]) and a usemantics for the logics determined for the 6 expansions of MK3 with only 2 as designated value (cf. [19]). It is obvious that it does not make sense to try and define a $\mathrm{u}$-semantics for the former logics, but we will show that the latter can equivalently be interpreted with a u-semantics and an o-semantics.

Concerning these semantics, there is another question that has to be remarked. Given a matrix M, as it is known, there are essentially two different ways of defining a consequence relation in $\mathrm{M}$ : a truth-preserving relation or a degree of truth-preserving relation (cf. Definition 7.1). In this sense, the $n$ valued Łukasiewicz logic $\mathrm{E} n(n=3, \ldots, \omega)$ is generally understood as the logic determined by the truth-preserving consequence relation defined in the matrix MŁn (cf. Definition 7.1). But Wójcicki has remarked that $€ n$ can also be viewed as the logic determined by the degree of truth-preserving consequence relation (cf. [26], §13). Actually, he goes as far as to affirm: "If not supplemented with a suitable epithet, Łukasiewicz logic is an ambiguous term" ([26], p. 42). Let us refer by $\mathrm{L} n^{1}$ and $\mathrm{L} n \leq$ to the former and the latter logic, respectively. Although both logics share, of course, the same set of valid formulas, they are fairly different, $\mathrm{L} n \leq$ being included in $\mathrm{L} n^{1}$ (cf. Definition 2.3) but not conversely. Well then, in our papers [17] and [19] referred to above, we have only considered truth-preserving consequence relations and their corresponding relations in BDsemantics (o-relations in [17] and u-relations in [19]). In the present paper, however, it will be shown that it is possible to define degree of truth-preserving 
consequence relations in 4 of the 6 natural implicative expansions of MK3 with only one designated value. Then, the corresponding equivalent o-relations and $\mathrm{u}$-relations will also be defined and the required strong soundness and completeness theorems will be proved. In this way, we define paraconsistent versions of four of the logics defined in [19] w.r.t. a truth-preserving consequence relation.

The present paper pursues previous work by the authors. In addition to [17] and [19] commented upon above, the following pages include and generalize to all logics determined by the 6 expansions of MK3 (with only one designated value) results of [18] and [16] where equivalent o-semantics and u-semantics are defined for Łuksiewicz's 3-valued logic Ł3 and positive 3-valued logic G3 expanded with a Łukasiewicz type negation, respectively.

In section 8 of the paper ("Concluding remarks"), we briefly comment upon some related work to the results in the present paper called to our attention by the referees of the JANCL.

The structure of the paper is as follows. In $\S 2$, we define some preliminary notions as, e.g., "Kleene's strong 3-valued matrix" or "natural conditional". In $\S 3$, six different Hilbert-type axiomatic systems are defined. It will be proved that each one of these systems is determined by one of the six natural implicative expansions of MK3 with only one designated value. The six systems are defined in a general and unified way taking Routley and Meyer's basic logic B (cf. [20], Chapter 4) as a reference point. In $\S 4$, equivalent o-semantics and u-semantics are provided for each one of the six systems defined in $\S 3$ and the soundness theorems are proved. In $\S 5$, we demonstrate a number of properties of theories built upon some or all of the six logics defined in $\S 3$. These properties are essentially used in the completeness proofs developed in $\S 6$. So far, only weak soundness and completeness theorems have been proved. In $\S 7$, truth-preserving and degree of truth-preserving consequence relations are defined together with their corresponding o-relations and/or u-relations. Then, strong soundness and completeness theorems are proved w.r.t. each one of these relations. The paper is ended in $\S 8$ with some remarks on the results obtained.

\section{Natural implicative expansions of MK3}

In this section, Kleene's strong 3-valued matrix and all its natural implicative expansions are recalled. Firstly, we state some preliminary definitions where we recall some basic notions as used in the present paper.

Definition 2.1 (Language) The propositional language consists of a denumerable set of propositional variables $p_{0}, p_{1}, \ldots, p_{n}, .$. , and some or all of the following connectives $\rightarrow$ (conditional), $\wedge$ (conjunction), $\vee$ (disjunction), $\neg$ (negation). The biconditional $(\leftrightarrow)$ and the set of wffs are defined in the customary way. A, B etc. are metalinguistic variables.

Definition 2.2 (Logics) A logic $L$ is a structure $\left(\mathcal{L}, \vdash_{L}\right)$ where $\mathcal{L}$ is a propositional language and $\vdash_{L}$ is a (proof-theoretical) consequence relation defined on 
$\mathcal{L}$ by a set of axioms and a set of rules of inference. The notions of 'proof' and 'theorem' are understood as it is customary in Hilbert-style axiomatic systems $\left(\Gamma \vdash_{L} A\right.$ means that $A$ is derivable in $L$ from the set of wffs $\Gamma$; and $\vdash_{L} A$ means that $A$ is a theorem of $L$ ).

Definition 2.3 (Extensions and expansions) Let $\mathcal{L}$ and $\mathcal{L}^{\prime}$ be two propositional languages. $\mathcal{L}^{\prime}$ is a strengthening of $\mathcal{L}$ if the set of wffs of $\mathcal{L}$ is a proper subset of the set of wffs of $\mathcal{L}^{\prime}$. Next, let $L$ and $L^{\prime}$ be two logics built upon the propositional languages $\mathcal{L}$ and $\mathcal{L}^{\prime}$, respectively. Moreover, suppose that all axioms of $L$ are theorems of $L^{\prime}$ and all primitive rules of inference of $L$ are provable in $L^{\prime}$. Then, $L^{\prime}$ is an extension of $L$ if $\mathcal{L}$ and $\mathcal{L}^{\prime}$ are the same propositional language; and $L^{\prime}$ is an expansion of $L$ if $\mathcal{L}^{\prime}$ is an strengthening of $\mathcal{L}$. An extension $L^{\prime}$ of $L$ is a proper extension if $L$ is not an extension of $L^{\prime}$.

Definition 2.4 (Logical matrix) $A$ (logical) matrix is a structure $(\mathcal{V}, D, \mathrm{~F})$ where (1) $\mathcal{V}$ is a (ordered) set of (truth) values; (2) $D$ is a non-empty proper subset of $\mathcal{V}$ (the set of designated values); and (3) $\mathrm{F}$ is the set of $n$-ary functions on $\mathcal{V}$ such that for each $n$-ary connective $c$ (of the propositional language in question), there is a function $f_{c} \in \mathrm{F}$ such that $\mathcal{V}^{n} \rightarrow \mathcal{V}$.

Definition 2.5 (M-interpretation, M-validity) Let $M$ be a matrix for ( $a$ propositional language) $\mathcal{L}$. An $M$-interpretation $I$ is a function from the set of all wfffs to $\mathcal{V}$ according to the functions in $\mathrm{F}$. Then, $\vDash_{M} A$ ( $A$ is M-valid; $A$ is valid in the matrix $M$ ) iff $I(A) \in D$ for all $M$-interpretations $I$.

We point out that the truth-preserving and degree of truth-preserving consequece relations definable in the matrices of interest in this paper are defined in Definition 7.1.

Definition 2.6 (Kleene's strong 3-valued matrix) The propositional language consists of the connectives $\wedge, \vee, \neg$. Kleene's strong 3-valued matrix, MK3 (our label), is the structure $(\mathcal{V}, D, F)$ where $(1) \mathcal{V}=\{0,1,2\}$ and it is ordered as shown in the following lattice

$$
\left.\right|_{0} ^{2}
$$

(2) $D=\{1,2\}$ or $D=\{2\}$; (3) $\mathrm{F}=\left\{f_{\wedge}, f_{\vee}, f_{\neg}\right\}$ where $f_{\wedge}$ and $f_{\vee}$ are defined as the glb (or lattice meet) and the lub (or lattice joint), respectively, $f_{\neg}$ is an involution with $f_{\neg}(2)=0, f_{\neg}(0)=2$ and $f_{\neg}(1)=1$. We display the tables for $\wedge, \vee$ and $\neg$ : 


\begin{tabular}{l|lll}
$\wedge$ & 0 & 1 & 2 \\
\hline 0 & 0 & 0 & 0 \\
1 & 0 & 1 & 1 \\
2 & 0 & 1 & 2
\end{tabular}

\begin{tabular}{c|ccc}
$\vee$ & 0 & 1 & 2 \\
\hline 0 & 0 & 1 & 2 \\
1 & 1 & 1 & 2 \\
2 & 2 & 2 & 2
\end{tabular}

\begin{tabular}{l|l}
$\neg$ & 0 \\
\hline 0 & 2 \\
1 & 1 \\
2 & 0
\end{tabular}

The notions of an MK3-interpretation and MK3-validity are defined according to the general Definition 2.5.

In what follows, all natural implicative expansions of MK3 are defined. Firstly, we define the notion of a natural conditional and the set of all natural conditionals in 3-valued matrices where $\mathcal{V}$ is as in MK3.

Following Tomova [24], we define "natural conditionals" as follows.

Definition 2.7 (Natural conditionals) Let $\mathcal{L}$ be a propositional language with $\rightarrow$ among its connectives and $M$ be a matrix for $\mathcal{L}$ where the values $x$ and $y$ represent the supremum and the infimum of $\mathcal{V}$. Then, an $f_{\rightarrow}$-function on $\mathcal{V}$ defines a natural conditional if the following conditions are satisfied:

1. $f_{\rightarrow}$ coincides with (the $f_{\rightarrow}$-function for) the classical conditional when restricted to the subset $\{x, y\}$ of $\mathcal{V}$.

2. $f \rightarrow$ satisfies Modus ponens, that is, for any $a, b \in \mathcal{V}$, if $a \rightarrow b \in D$ and $a \in D$, then $b \in D$.

3. For any $a, b \in \mathcal{V}, a \rightarrow b \in D$ if $a \leq b$.

Proposition 2.8 (Natural conditionals in 3-valued matrices) (a) Two designated values: Let $\mathcal{L}$ be a propositional language and $M$ be a 3-valued matrix where $\mathcal{V}$ is defined exactly as in $M K 3$ and $D=\{1,2\}$. Now, consider the $24 f_{\rightarrow}$ functions defined in the following general table:

$T I$

\begin{tabular}{l|lll}
$\rightarrow$ & 0 & 1 & 2 \\
\hline 0 & 2 & $a_{1}$ & 2 \\
1 & 0 & $a_{2}$ & $a_{3}$ \\
2 & 0 & $b_{1}$ & 2
\end{tabular}

where $a_{i}(1 \leq i \leq 3) \in\{1,2\}$ and $b_{1} \in\{0,1,2\}$. The set of functions contained in $T I$ is the set of all natural conditionals definable in $M$.

(b) One designated value: Now, let $M$ and $\mathcal{V}$ be as above but $D=\{2\}$. Consider the $6 f \rightarrow$ functions in the following general table:

TII

\begin{tabular}{l|lll}
$\rightarrow$ & 0 & 1 & 2 \\
\hline 0 & 2 & 2 & 2 \\
1 & $a$ & 2 & 2 \\
2 & 0 & $b$ & 2
\end{tabular}

where $a \in\{0,1,2\}$ and $b \in\{0,1\}$. The set of functions contained in TII is the set of all natural conditionals definable in $M$. 
Proof. It is obvious (cf. [17], [19]. These tables can be found in [23] and [25], in addition to [24] (we owe this remark to a referee of the JANCL).

Next, all natural implicative expansions of MK3 are defined.

Definition 2.9 (Natural implicative expansions of MK3) (a) Two designated values: There are exactly 24 natural implicative expansions of MK3, which are defined as follows. Each one of them is the structure $(\mathcal{V}, D, F)$ where $\mathcal{V}, D, f_{\wedge}, f_{\vee}$ and $f_{\neg}$ are defined exactly as in MK3 (cf. Definition 2.6, with $D=\{1,2\}$ ), whereas $f_{\rightarrow}$ is defined according to one of the tables in TI. (b) Only one designated value: There are exactly 6 natural implicative expansions of $M K 3$, which are defined as follows. Each one of them is the structure $(\mathcal{V}, D$, $F$ ) where $\mathcal{V}, D, f_{\wedge}, f_{\vee}$ and $f_{\neg}$ are defined exactly as in MK3 (cf. Definition 2.6, with $D=\{2\}$ ), whereas $f_{\rightarrow}$ is defined according to one of the the tables in TII. The notions of M-interpretation and M-validity are understood according to the general Definition 2.5.

The aim of this paper is to provide equivalent underdetermined and overdetermined BD-semantics for the logics determined by the expansions of MK3 with only one designated value defined above in Definition 2.9 (cf. Definition 7.4). For the reader's convenience, we display the six tables (designated values are starred):

t1

\begin{tabular}{l|lll}
$\rightarrow$ & 0 & 1 & 2 \\
\hline 0 & 2 & 2 & 2 \\
1 & 0 & 2 & 2 \\
$*_{2}$ & 0 & 0 & 2
\end{tabular}

t4

\begin{tabular}{l|lll}
$\rightarrow$ & 0 & 1 & 2 \\
\hline 0 & 2 & 2 & 2 \\
1 & 1 & 2 & 2 \\
$*_{2}$ & 0 & 0 & 2
\end{tabular}

t2

\begin{tabular}{l|lll}
$\rightarrow$ & 0 & 1 & 2 \\
\hline 0 & 2 & 2 & 2 \\
1 & 1 & 2 & 2 \\
$* 2$ & 0 & 1 & 2
\end{tabular}

t5

\begin{tabular}{l|lll}
$\rightarrow$ & 0 & 1 & 2 \\
\hline 0 & 2 & 2 & 2 \\
1 & 2 & 2 & 2 \\
$*_{2}$ & 0 & 1 & 2
\end{tabular}

t3

\begin{tabular}{l|lll}
$\rightarrow$ & 0 & 1 & 2 \\
\hline 0 & 2 & 2 & 2 \\
1 & 0 & 2 & 2 \\
$*_{2}$ & 0 & 1 & 2
\end{tabular}

t6

\begin{tabular}{l|lll}
$\rightarrow$ & 0 & 1 & 2 \\
\hline 0 & 2 & 2 & 2 \\
1 & 2 & 2 & 2 \\
$*_{2}$ & 0 & 0 & 2
\end{tabular}

We shall refer by Mt $i(1 \leq i \leq 6)$ to the expansion of MK3 defined upon the table $t i$; and by Lt $i(1 \leq i \leq 6)$ to the logic determined by Mti.

We note that Lt2 is a logic equivalent to Lukasiewicz's 3-valued logic L3. Lt3 is a logic equivalent to G3 ${ }_{\mathrm{L}}$ (cf. [16]) and Lt5 is treated in [12] (cf. p. 65). But the logics determined by Mt1, Mt4 and Mt6 have not been considered in the literature, as far as we know. (However, see section 8.)

\section{The Lt $i$-logics}

We shall generally refer by the term Lti-logics to the logics determined by the Mti-implicative expansions of MK3 defined in Definition 2.9(b). We shall axiomatize the Lt $i$-logics in a general and unified way. We use Routley and Meyer's basic logic B, which is defined below (cf. [20], Chapter 4). 
Definition 3.1 (The logic B) Routley and Meyer's basic logic B is axiomatized with the following axioms and rules of inference.

Axioms:

$$
\begin{aligned}
& \text { A1. }(A \wedge B) \rightarrow A /(A \wedge B) \rightarrow B \\
& \text { A2. }[(A \rightarrow B) \wedge(A \rightarrow C)] \rightarrow[A \rightarrow(B \wedge C)] \\
& \text { A3. } A \rightarrow(A \vee B) / B \rightarrow(A \vee B) \\
& \text { A4. }[(A \rightarrow C) \wedge(B \rightarrow C)] \rightarrow[(A \vee B) \rightarrow C] \\
& \text { A5. }[A \wedge(B \vee C)] \rightarrow[(A \wedge B) \vee(A \wedge C)] \\
& \text { A6. } A \rightarrow \neg \neg A \\
& \text { A\%. } \neg \neg A \rightarrow A
\end{aligned}
$$

Rules of inference:

$$
\begin{aligned}
& \text { Adjunction (Adj): } A \& B \Rightarrow A \wedge B \\
& \text { Modus Ponens (MP): } A \rightarrow B \& A \Rightarrow B \\
& \text { Suffixing (Suf): }(A \rightarrow B) \Rightarrow(B \rightarrow C) \rightarrow(A \rightarrow C) \\
& \text { Prefixing (Pref): }(B \rightarrow C) \Rightarrow(A \rightarrow B) \rightarrow(A \rightarrow C) \\
& \text { Contraposition (Con): }(A \rightarrow B) \Rightarrow \neg B \rightarrow \neg A
\end{aligned}
$$

We recall some theorems of B.

Proposition 3.2 (Some theorems of B) The following theorems are provable in $B$ (cf. [20], Chapter 4): (T1) $A \rightarrow A$; (T2) $\neg(A \vee B) \leftrightarrow(\neg A \wedge \neg B)$; (T3) $\neg(A \wedge B) \leftrightarrow(\neg A \vee \neg B)$.

We note the following remark.

Remark 3.3 (The positive fragment of B) The positive (i.e., negationless) fragment of B, $B_{+}$, is axiomatized with T1, A1-A5, Adj, MP, Suf and Pref (cf. [20], Chapter 4).

Next, we define the logic $b^{3}$, which is included in each one of the Lt $i$-logics (the label $\mathrm{b}^{3}$ is intended to abbreviate "basic logic contained in the six Lt $i$ logics"). Then, two extensions of $b^{3}, b_{1}^{3}$ and $b_{2}^{3}$, are defined. Lt1 through Lt4 are extensions of the former, whereas Lt5 and Lt6 include the latter.

Definition 3.4 (The logic $\mathbf{b}^{3}$ ) The basic logic $b^{3}$ is axiomatized with A1-A7, $(A \wedge \neg A) \rightarrow(B \vee \neg B)$ (A8), Adj, MP, Suf and Pref. That is, $b^{3}$ is the result of adding A6, A7 and A8 to $B_{+}$. Or, from another point of view, it is $B$ minus Con and plus $A 8$. 
Definition 3.5 (The logic $\mathbf{b}_{1}^{3}$ ) The logic $b_{1}^{3}$ is the result of adding the rule Veq and the following axioms to B plus A8 (or $b^{3}$ plus Con) ("Verum e quodlibet" means "A true proposition follows from any proposition"):

Axioms:

$$
\begin{aligned}
& \text { A9. } \neg A \rightarrow[A \vee(A \rightarrow B)] \\
& \text { A10. } B \rightarrow[\neg B \vee(A \rightarrow B)] \\
& \text { A11. }[\neg(A \rightarrow B) \wedge(\neg A \wedge B)] \rightarrow C \\
& \text { A12. }[(A \rightarrow B) \wedge A] \rightarrow(\neg A \vee B) \\
& \text { A13. }[(A \rightarrow B) \wedge \neg B] \rightarrow(\neg A \vee B)
\end{aligned}
$$

Rule: "Verum e quodlibet" (Veq). $A \Rightarrow B \rightarrow A$

In the following proposition, some theorems and a rule of $b_{1}^{3}$ are remarked.

Proposition 3.6 (Some theorems of $\mathbf{b}_{1}^{3}$ ) The rule Efq and the following theorems are provable in $b_{1}^{3}$ ("E falso quodlibet" means "Any proposition follows from a false proposition"): (T4) $[\neg(A \rightarrow B) \wedge \neg A] \rightarrow A ;(T 5)[\neg(A \rightarrow B) \wedge B] \rightarrow$ $\neg B ;(T 6)(A \vee \neg B) \vee(A \rightarrow B) ;(T 7)(A \wedge \neg B) \rightarrow[\neg(A \rightarrow B) \vee \neg A]$; (T8) $(A \wedge \neg B) \rightarrow[\neg(A \rightarrow B) \vee B]$; "E falso quodlibet" (Efq). $A \Rightarrow \neg A \rightarrow B$.

Proof. T4, T5, T7 and T8 are easily derivable from B and A9, A10, A12 and A13, respectively (by using Con, A6, A7 and the De Morgan laws T2 and T3). Then, T6 is derivable by Con, A11, A6, A7 and the De Morgan laws, and, finally, Efq follows by Veq, Con and A7.

Next, the Lt $i$-logics Lt1 through Lt4 are defined as extensions of $b_{1}^{3}$.

Definition 3.7 (The Lti-logics Lt1-Lt4) The Lti-logics Lt1 through Lt4 are the result of adding the following axioms to $b_{1}^{3}$.

Lt1:

$$
\begin{aligned}
& \text { A14. }[(A \rightarrow B) \wedge A] \rightarrow B \\
& A 15 .[(A \rightarrow B) \wedge \neg B] \rightarrow \neg A
\end{aligned}
$$

Lt2:

$$
\begin{aligned}
& \text { A16. } A \rightarrow(B \rightarrow A) \\
& \text { A17. } \neg A \rightarrow(A \rightarrow B)
\end{aligned}
$$

Lt3:

$$
\begin{aligned}
& \text { A14. }[(A \rightarrow B) \wedge A] \rightarrow B \\
& \text { A16. } A \rightarrow(B \rightarrow A)
\end{aligned}
$$

Lt4:

A15. $[(A \rightarrow B) \wedge \neg B] \rightarrow \neg A$
A17. $\neg A \rightarrow(A \rightarrow B)$ 
Some theorems of Lt1-Lt4 are pointed out below.

Proposition 3.8 (Some theorems of Lt1-Lt4) The following theorems are proved in Lt1-Lt4: (T9) $A \rightarrow[B \vee \neg(A \rightarrow B)]$ in Lt1 and Lt4; (T10) $\neg B \rightarrow$ $[\neg A \vee \neg(A \rightarrow B)]$ in Lt1 and Lt3; (T11) $\neg(A \rightarrow B) \rightarrow(A \wedge \neg B)$ in Lt2; (T12) $\neg(A \rightarrow B) \rightarrow \neg B$ in Lt2 and Lt3; (T13) $\neg(A \rightarrow B) \rightarrow A$ in Lt2 and Lt4.

Proof. T9 (resp., T10): by A15 (resp., A14), Con and T3, T6, T7; T12: by Con and A16; T13: by Con, A17 and A7; T11: by T12 and T13.

Definition 3.9 (The logic $\mathbf{b}_{2}^{3}$ ) The logic $b_{2}^{3}$ is the result of adding the following axioms to $b^{3}$ :

$$
\begin{aligned}
& \text { A14. }[(A \rightarrow B) \wedge A] \rightarrow B \\
& \text { A16. } A \rightarrow(B \rightarrow A) \\
& \text { A17. } \neg A \rightarrow(A \rightarrow B) \\
& \text { A18. } A \vee(A \rightarrow B) \\
& \text { A19. } \neg(A \vee B) \leftrightarrow(\neg A \wedge \neg B) \\
& \text { A20. } \neg(A \wedge B) \leftrightarrow(\neg A \vee \neg B) \\
& \text { A21. }(A \wedge \neg B) \rightarrow[\neg A \vee \neg(A \rightarrow B)] \\
& \text { A22. }[\neg(A \rightarrow B) \wedge(\neg A \vee B)] \rightarrow C
\end{aligned}
$$

Next, Lt5 and Lt6 are defined as extensions of $\mathrm{b}_{2}^{3}$.

Definition 3.10 (The logics Lt5 and Lt6) The logics Lt5 and Lt6 are the result of adding the following axioms to $b_{2}^{3}$ :

Lt5:

$$
\text { A23. } \neg(A \rightarrow B) \leftrightarrow(A \wedge \neg B)
$$

Lt6:

$$
\begin{aligned}
& \text { A24. } A \rightarrow[B \vee \neg(A \rightarrow B)] \\
& \text { A25. }[(A \rightarrow B) \wedge(A \wedge \neg B)] \rightarrow C
\end{aligned}
$$

Given the axiomatizations defined above, it is not difficult to provide more conspicuous formulations of the Lti-logics — cf. [17], §9 and [19], §10.

\section{BD-semantics for the Lt $i$-logics}

In this section, we define a BD-semantics for each one of the Lti-logics. We will define two types of models, underdetermined models (u-models) and overdetermined models (o-models). It will be proved that each Lti-logic is (weakly) sound (this section) and weakly complete (section 6) w.r.t. both its corresponding u-models and o-models. Strong soundness and completeness are treated in section 7 . 
In the first place, we define the general notions of a u-model and an o-model. Then, uLt $i$-models and oLt $i$-models are defined. By a semantics, we mean a class of models together with the accompanying notion of validity. Thus, for instance, an oLt2-semantics is formed by the class of all oLt2-models and the annexed definition of oLt2-validity (cf. Definitions 4.5 and 4.6 below).

Definition 4.1 (u-models) An underdetermined model (u-model) is a structure $(K, I)$ where (i) $K=\{\{T\},\{F\}, \emptyset\}$ and (ii) $I$ is a u-interpretation from the set of all wffs to $K$, this notion being defined according to the following conditions for each propositional variable $p$ and wffs $A, B:$ (1) $I(p) \in K$; (2a) $T \in I(\neg A)$ iff $F \in I(A)$; (2b) $F \in I(\neg A)$ iff $T \in I(A)$; (3a) $T \in I(A \wedge B)$ iff $T \in I(A)$ and $T \in I(B)$; (3b) $F \in I(A \wedge B)$ iff $F \in I(A)$ or $F \in I(B)$; (4a) $T \in I(A \vee B)$ iff $T \in I(A)$ or $T \in I(B)$; (4b) $F \in I(A \vee B)$ iff $F \in I(A)$ and $F \in I(B)$; (5a) there are two possibilities for assigning $T$ to conditionals: (5a1) $T \in I(A \rightarrow B)$ iff $F \in I(A)$ or $T \in I(B)$ or $[T \notin I(A)$ and $F \notin I(B)]$; (5a2) $T \in I(A \rightarrow B)$ iff $T \notin I(A)$ or $T \in I(B)$; (5b) there are four possibilities for assigning $F$ to conditionals: (5b1) $F \in I(A \rightarrow B)$ iff $[T \in I(A)$ and $T \notin I(B)]$ or $[F \notin I(A)$ and $F \in I(B)]$; (5b2) $F \in I(A \rightarrow B)$ iff $T \in I(A)$ and $F \in I(B)$; (5b3) $F \in I(A \rightarrow B)$ iff $F \notin I(A)$ and $F \in I(B)$; and (5b4) $F \in I(A \rightarrow B)$ iff $T \in I(A)$ and $T \notin I(B)$.

Then, underdetermined Lt $i$-models (uLt $i$-models) are defined as follows.

Definition 4.2 (uLt $i$-models) For all $i(1 \leq i \leq 4)$, uLti-models are $u$-models with clauses (1)-(4b), $5 a 1$ and 5bi. Then, uLt5-models are $u$-models with clauses 5a2 and 5b2; and uLt6-models are u-models with clauses 5a2 and $5 b 4$.

Definition 4.3 (uLt $i$-validity) Let $M$ be a uLti-model $(1 \leq i \leq 6)$. A is true in $M$ (in symbols, $\vDash_{M}^{u} A$ ) iff $T \in I(A)$ ( $I$ is the uLti-interpretation defined in $M)$; and $A$ is valid in uLti-semantics (in symbols, $\vDash_{L t i}^{u} A$ ) iff $A$ is true in all uLti-models $M$.

Next, o-models and o-validity are defined.

Definition 4.4 (o-models) An o-model is a structure $(K, I)$ where (i) $K=$ $\{\{T\},\{F\},\{T, F\}\}$ and (ii) $I$ is an o-interpretation from the set of all wffs to $K$, this notion being defined according to clauses 1, 2a, 2b, 3a, 3b, 4a and $4 b$ in Definition 4.1 for each propositional variable $p$ and wffs $A, B$, while the clauses for the conditional are as follows. (5a) There are four possibilities for assigning $T$ to conditionals: (5a1) $T \in I(A \rightarrow B)$ iff $T \notin I(A)$ or $F \notin I(B)$ or $[F \in I(A)$ and $T \in I(B)]$; (5a2) $T \in I(A \rightarrow B)$ iff $F \in I(A)$ or $T \in I(B)$; (5a3) $T \in I(A \rightarrow B)$ iff $T \notin I(A)$ or $T \in I(B)$; $\left(5 a_{4}\right) T \in I(A \rightarrow B)$ iff $F \in I(A)$ or $F \notin I(B) ;(5 b)$ there are two possibilities for assigning $F$ to conditionals: (5b1) $F \in I(A \rightarrow B)$ iff $[T \in I(A)$ and $T \notin I(B)]$ or $[F \notin I(A)$ and $F \in I(B)]$; (5b2) $F \in I(A \rightarrow B)$ iff $F \notin I(A)$ and $F \in I(B)$.

Then, overdetermined Lt $i$-models (oLt $i$-models) are defined as follows. 
Definition 4.5 (oLt $i$-models) For all $i(1 \leq i \leq 4)$, oLti-models are o-models with clauses (1)-(4b), 5ai and 5b1. Then, oLt5-models are o-models with clauses 5a2 and 5b2, and oLt6-models are o-models with clauses 5a4 and 5b2.

Definition 4.6 (oLt $i$-validity) Let $M$ be an oLti-model $(1 \leq i \leq 4)$. $A$ is true in $M$ (in symbols, $\models_{M}^{o} A$ ) iff $F \notin I(A)$ ( $I$ is the oLti-interpretation defined in $M$ ); and $A$ is valid in oLti-semantics (in symbols, $\vDash_{L t i}^{o} A$ ) iff $A$ is true in all oLti-models $M$.

We note the following remark:

Remark 4.7 (Alternative interpretations of the conditional) Of course, there are other possibilities for assigning $F$ (resp., $T$ ) to conditionals in u-models (resp., o-models) (cf. [17] and [19]). Here we have restricted ourselves to the conditions of interest for interpreting the logics Lt1 through Lt6.

Next, it is proved that the valuation relation $\vDash_{\mathrm{Mti}}$ (cf. Definition 2.9) and the relations $\models_{\mathrm{Lt} i}^{\mathrm{u}}$ and $\models_{\mathrm{Lt} i}^{\mathrm{o}}$ just defined are coextensive. Then, (weak) soundness of each Lti-logic w.r.t. the three valuation relations will follow immediately.

Firstly, the notion of corresponding interpretation is defined and a proposition on corresponding interpretations is proved.

Definition 4.8 (Corresponding interpretations) Let I be a u-interpretation. The corresponding Mti-interpretation to $I, I^{\prime}$, is defined as follows. For each propositional variable, we set: (1) $I(p)=\{T\}$ iff $I^{\prime}(p)=2$; (2) $I(p)=\emptyset$ iff $I^{\prime}(p)=1$; and (3) $I(p)=\{F\}$ iff $I^{\prime}(p)=0$. Then, complex wffs are evaluated as stated in Definition 2.9. Conversely, given an Mti-interpretation I, the corresponding u-interpretation, $I^{\prime}$, is defined similarly w.r.t. propositional variables, whereas complex wffs are, of course, evaluated according to clauses 2-5 in Definition 4.1.

On the other hand, corresponding Mti-interpretations (resp., o-interpretations) to o-interpretations (resp., Mti-interpretations) are defined similarly, except that now complex wffs are evaluated with Definition 2.9 (in the case of Mtiinterpretations) and Definition 4.4 (in the case of o-interpretations), while propositional variables are interpreted as follows ( $I$ stands for the o-interpretation and $I^{\prime}$ for the Mti-interpretation in question): (1) $I(p)=\{T\}$ iff $I^{\prime}(p)=2$; (2) $I(p)=\{T, F\}$ iff $I^{\prime}(p)=1$; and (3) $I(p)=\{F\}$ iff $I^{\prime}(p)=0$.

Proposition 4.9 (Equivalence of corresponding interpretations) (I) Let $I$ be a u-interpretation (resp., Mti-interpretation) and $I^{\prime}$ be the Mti-interpretation (resp., u-interpretation) corresponding to $I$. Then, for each wff $A$, we have: (1) $I(A)=\{T\}$ iff $I^{\prime}(A)=2$; (2) $I(A)=\emptyset$ iff $I^{\prime}(A)=1$; and (3) $I(A)=\{F\}$ iff $I^{\prime}(A)=0$.

(II) Let I be an o-interpretation (resp., Mti-interpretation) and $I^{\prime}$ be the Mti-interpretation (resp., o-interpretation) corresponding to I. Then, for each wff A, we have: (1) $I(A)=\{T\}$ iff $I^{\prime}(A)=2$; (2) $I(A)=\{T, F\}$ iff $I^{\prime}(A)=1$; and (3) $I(A)=\{F\}$ iff $I^{\prime}(A)=0$. 
Proof. By an easy induction on the length of $A$.

Proposition 4.10 (Coextensiveness of $\vDash_{\mathrm{Mt} i}, \vDash_{\mathrm{Lt} i}^{\mathrm{u}}$ and $\left.\models_{\mathrm{Lt} i}^{\mathrm{o}}\right)$ For any $i(1 \leq$ $i \leq 6)$ and wff $A,(1) \vDash_{M t i} A$ iff $\vDash_{L t i}^{u} A$; (2) $\vDash_{M t i} A$ iff $\models_{L t i}^{o} A$.

Proof. Immediate by Definitions 2.9, 4.3, 4.6 and Proposition 4.9.

Now, we can prove (weak) soundness.

Theorem 4.11 (Soundness of Lt $i$ w.r.t. $\left.\vDash_{\mathrm{Mt} i}\right)$ For any $i(1 \leq i \leq 6)$ and wff $A$, if $\vdash_{L t i} A$, then $\vDash_{M t i} A$.

Proof. Given a particular logic Lti, it is easy to check that the rules Adj, MP, Suf, Pref, Veq - and Con in the case of Lt1-Lt4 - preserve Mti-validity (if the premises of each rule are MLti-valid, so is its conclusion). On the other hand, the axioms of Lt $i$ are assigned the value 2 by any Mt $i$-interpretation $I$. Consequently, if $\vdash_{\mathrm{Lt} i} A$, then $\vdash_{\mathrm{Mt} i} A$. (In case a tester is needed, the reader can use that in [9].)

Finally, we have the following corollary establishing soundness w.r.t. $\vDash_{\mathrm{Lt} i}^{\mathrm{u}}$ and $\models_{\mathrm{Lt} i}^{\mathrm{o}}$.

Corollary 4.12 (Soundness w.r.t. $\models_{\mathrm{Lt} i}^{\mathbf{u}}$ and $\left.\models_{\mathrm{Lt} i}^{\mathbf{o}}\right)$ For any $i(1 \leq i \leq 6)$ and $w f f A$, if $\vdash_{L t i} A$, then (1) $\vDash_{L t i}^{u} A$ and (2) $\vDash_{L t i}^{o} A$.

Proof. Immediate by Proposition 4.10 and Theorem 4.11.

\section{Theories and their properties}

In what follows, we prove some properties of $\mathrm{b}^{3}$ and its extensions or expansions. These properties will be used in the completeness proofs to follow. We begin by defining the notion of $a b^{3}$-theory and the classes of $b^{3}$-theories of interest in the present paper (by EL, we generally refer to an extension - or an expansion, as the case may be - of the logic L; cf. Definition 2.3).

Definition 5.1 ( $\mathbf{E b}^{3}$-theories) Let $L$ be an $E b^{3}$-logic. An L-theory is a set of wffs closed under Adjunction (Adj) and provable L-entailment (L-ent). That is, $\mathcal{T}$ is an L-theory if whenever $A, B \in \mathcal{T}$, then $A \wedge B \in \mathcal{T}$; and if whenever $A \rightarrow B$ is a theorem of $L$ and $A \in \mathcal{T}$, then $B \in \mathcal{T}$ (L-ent).

Definition 5.2 (Classes of $\mathbf{E b}^{3}$-theories) Let $L$ be an $E b^{3}$-logic and $\mathcal{T}$ an L-theory. We set: (1) $\mathcal{T}$ is prime iff whenever $A \vee B \in \mathcal{T}$, then $A \in \mathcal{T}$ or $B \in \mathcal{T}$; (2) $\mathcal{T}$ is regular iff $\mathcal{T}$ contains all theorems of $L$; (3) $\mathcal{T}$ is complete iff for any wff $A, A \in \mathcal{T}$ or $\neg A \in \mathcal{T}$; (4) $\mathcal{T}$ is trivial iff it contains all wffs; (5) $\mathcal{T}$ is a-consistent (consistent in an absolute sense) iff $\mathcal{T}$ is not trivial. Finally, (6) $\mathcal{T}$ is consistent iff it is not inconsistent ( $\mathcal{T}$ is inconsistent iff $A \wedge \neg A \in \mathcal{T}$ for some wff $A$ ).

Then, we have: 
Proposition $5.3\left(\mathrm{~Eb}^{3}\right.$-theories and double negation) Let $L$ be an $E b^{3}$-logic and $\mathcal{T}$ an L-theory. Then, $A \in \mathcal{T}$ iff $\neg \neg A \in \mathcal{T}$.

Proof. Immediate by A6 and A7, given that $\mathcal{T}$ is closed under L-ent.

Proposition 5.4 (Conjunction and disjunction in prime $\mathbf{E b}^{3}$-theories) Let $L$ be an $E b^{3}$-logic and $\mathcal{T}$ be a prime L-theory. Then, (1) $A \wedge B \in \mathcal{T}$ iff $A \in \mathcal{T}$ and $B \in \mathcal{T}$; (2) $\neg(A \wedge B) \in \mathcal{T} \quad$ iff $\neg A \in \mathcal{T}$ or $\neg B \in \mathcal{T}$; (3) $A \vee B \in \mathcal{T}$ iff $A \in \mathcal{T}$ or $B \in \mathcal{T}$; (4) $\neg(A \vee B) \in \mathcal{T} \quad$ iff $\neg A \in \mathcal{T}$ and $\neg B \in \mathcal{T}$.

Proof. Immediate by $\mathrm{B}_{+}$(cf. Remark 3.3) and the De Morgan laws (cf. T2 and T3 - Proposition 3.2; A19 and A20 - Definition 3.9).

Next, we remark some fundamental properties of the conditional in $\mathrm{Eb}_{1}^{3}$ logics and $\mathrm{Eb}_{2}^{3}$-logics. We consider prime, complete and a-consistent $\mathrm{Eb}_{1}^{3}$ theories (resp., $\mathrm{Eb}_{2}^{3}$-theories) as well as prime, regular and consistent $\mathrm{Eb}_{1^{-}}^{3}$ theories (resp., $\mathrm{Eb}_{2}^{3}$-theories).

Proposition 5.5 ( $\rightarrow$ in prime, complete, a-consistent $\mathbf{E b}_{1}^{3}$-theories) Let $L$ be an $E b_{1}^{3}$-logic and $\mathcal{T}$ be a prime, complete and a-consistent $L$-theory. Then, $\neg(A \rightarrow B) \in \mathcal{T} \quad$ iff $[A \in \mathcal{T}$ and $B \notin \mathcal{T}]$ or $[\neg A \notin \mathcal{T}$ and $\neg B \in \mathcal{T}]$.

Proof. From left to right $(\Rightarrow)$ : Suppose $(1) \neg(A \rightarrow B) \in \mathcal{T}$ and, for reductio, that one of the next four alternatives follows. (2) $A \notin \mathcal{T} \quad \& \quad \neg A \in \mathcal{T}$; (3) $A \notin \mathcal{T} \& \neg B \notin \mathcal{T}$; (4) $B \in \mathcal{T} \quad \& \neg A \in \mathcal{T}$ or $(5) B \in \mathcal{T} \& \neg B \notin \mathcal{T}$. But these four alternatives are impossible: (2) by $[\neg(A \rightarrow B) \wedge \neg A] \rightarrow A$ (T4); and (5) by $[\neg(A \rightarrow B) \wedge B] \rightarrow \neg B$ (T5). Then, the impossibility of (3) and (4) are proved as follows. Let $C$ be an arbitrary wff. By (4) and $[\neg(A \rightarrow B) \wedge(\neg A \wedge B)] \rightarrow C$, we get (A11) $C \in \mathcal{T}$, contradicting the a-consistency of $\mathcal{T}$. Finally, given (3), we have $\neg A \in \mathcal{T}$ and $B \in \mathcal{T}$, since $\mathcal{T}$ is complete. Then, the impossibility of (3) is shown similarly as that of (4).

From right to left $(\Leftarrow)$ : Suppose $(1) A \in \mathcal{T}$ and $B \notin \mathcal{T}$. By completeness of $\mathcal{T}$, we have $\neg B \in \mathcal{T}$. Then, $\neg(A \rightarrow B) \in \mathcal{T}$ follows by $(A \wedge \neg B) \rightarrow[\neg(A \rightarrow$ $B) \vee B](\mathrm{T} 8)$ and the primeness of $\mathcal{T}$. Suppose now $(2) \neg A \notin \mathcal{T}$ and $\neg B \in \mathcal{T}$. Then, $\neg(A \rightarrow B) \in \mathcal{T}$ follows similarly as in the precedent case by using now $(A \wedge \neg B) \rightarrow[\neg(A \rightarrow B) \vee \neg A](\mathrm{T} 7)$.

Proposition 5.6 ( $\rightarrow$ in prime, regular, consistent $\mathbf{E b}_{1}^{3}$-theories) Let $L$ be an $E b_{1}^{3}$-logic and $\mathcal{T}$ be a prime, regular and consistent L-theory. Then, $A \rightarrow$ $B \in \mathcal{T} \quad$ iff $\neg A \in \mathcal{T}$ or $B \in \mathcal{T}$ or $(A \notin \mathcal{T}$ and $\neg B \notin \mathcal{T})$.

Proof. ( $\Rightarrow$ ): Suppose (1) $A \rightarrow B \in \mathcal{T}$ and, for reductio, (2) $\neg A \notin \mathcal{T} \& B \notin \mathcal{T}$ $\& A \in \mathcal{T}$ or $(3) \neg A \notin \mathcal{T} \& B \notin \mathcal{T} \& \neg B \in \mathcal{T}$. But 2 and 3 are impossible by A12 and A13, respectively, since $\mathcal{T}$ is prime.

$(\Leftarrow)$ : (1) Suppose $\neg A \in \mathcal{T}$. We get $A \notin \mathcal{T}$ since $\mathcal{T}$ is consistent. Then $A \rightarrow B \in \mathcal{T}$ follows by $\neg A \rightarrow[A \vee(A \rightarrow B)]$ (A9) and primeness of $\mathcal{T}$. (2) Suppose $B \in \mathcal{T}$. By consistency of $\mathcal{T}$, we have $\neg B \notin \mathcal{T}$. Then $A \rightarrow B \in \mathcal{T}$ is derivable similarly as in case (1) by using now $B \rightarrow[\neg B \vee(A \rightarrow B)]$ (A10). (3) 
Suppose $A \notin \mathcal{T}$ and $\neg B \notin \mathcal{T}$. As $\mathcal{T}$ is regular, $(A \vee \neg B) \vee(A \rightarrow B) \in \mathcal{T}$ by T6. Then, we have $A \rightarrow B \in \mathcal{T}$ by primeness of $\mathcal{T}$.

Proposition 5.7 ( $\rightarrow$ in prime, complete, a-consistent $\mathbf{E b}_{2}^{3}$-theories) Let $L$ be an $E b_{2}^{3}$-logic and $\mathcal{T}$ be a prime, complete and a-consistent $L$-theory. Then, $\neg(A \rightarrow B) \in \mathcal{T} \quad$ iff $\neg A \notin \mathcal{T}$ and $\neg B \in \mathcal{T}$.

Proof. Similar to those of Propositions 5.5 and 5.6, by using now $(A \wedge \neg B) \rightarrow$ $[\neg A \vee \neg(A \rightarrow B)](\mathrm{A} 21)$ and $[\neg(A \rightarrow B) \wedge(\neg A \vee B)] \rightarrow C(\mathrm{~A} 22)$.

Proposition 5.8 ( $\rightarrow$ in prime, regular, consistent $\mathbf{E b}_{2}^{3}$-theories) Let $L$ be an $E b_{2}^{3}$-logic and $\mathcal{T}$ be a prime, regular and consistent L-theory. Then, $A \rightarrow$ $B \in \mathcal{T}$ iff $A \notin \mathcal{T}$ or $B \in \mathcal{T}$.

Proof. Similar to those of Propositions 5.5, 5.6 and 5.7 by using now [ $(A \rightarrow$ $B) \wedge A] \rightarrow B$ (A14), $A \rightarrow(B \rightarrow A)(\mathrm{A} 16)$ and $A \vee(A \rightarrow B)$ (A18).

We prove an additional property of $\mathrm{Eb}^{3}$-logics and then the primeness lemma.

Proposition 5.9 (Consistency or completeness) Let $L$ be an $E b^{3}$-logic and $\mathcal{T}$ be a prime L-theory. If $\mathcal{T}$ is inconsistent, then it is complete.

Proof. Immediate by A8.

Lemma 5.10 (Primeness) Let $L$ be an $E b^{3}$-logic, $\mathcal{T}$ an L-theory and $A$ a formula such that $A \notin \mathcal{T}$. Then, there is a prime $L$-theory $\mathcal{T}^{\prime}$ such that $\mathcal{T} \subseteq \mathcal{T}^{\prime}$ and $A \notin \mathcal{T}^{\prime}$.

Proof. We extend $\mathcal{T}$ to a maximal theory $\mathcal{T}^{\prime}$ such that $A \notin \mathcal{T}^{\prime}$. Then, it is easy to show that $\mathcal{T}^{\prime}$ is prime (cf., for example, the proof of Lemma 5.13 in [17]. This proof holds for any logic L that includes the positive fragment of Anderson and Belnap's First Degree Entailment logic, FDE + (cf. [1], §15.2), provided that L-theories are defined similarly as $\mathrm{Eb}^{3}$-theories, that is, as sets of formulas closed under L-ent and Adj).

In Propositions 5.5, 5.6, 5.7 and 5.8, we have set down some fundamental properties of the conditional in $\mathrm{Eb}^{3}$-logics, $\mathrm{Eb}_{1}^{3}$-logics and $\mathrm{Eb}_{2}^{3}$-logics. In what follows, the essential properties of the conditional in the $\mathrm{Eb}_{1}^{3}$ - and $\mathrm{Eb}_{2}^{3}$-logics Lt1 through Lt6 are added to the aforementioned (and more general) properties.

Proposition 5.11 ( $\rightarrow$ in prime, complete, a-consistent Lt $i$-theories) Let $L$ be an ELti-logic where Lti will refer in each case to one of the extensions of $b^{3}$ displayed in Definitions 3.7 and 3.10. And let $\mathcal{T}$ be a prime, complete and a-consistent L-theory. We have: ELt1-logics: $A \rightarrow B \in \mathcal{T}$ iff $A \notin \mathcal{T}$ or $\neg B \notin \mathcal{T}$ or $[\neg A \in \mathcal{T}$ and $B \in \mathcal{T}]$; ELt2-logics: $A \rightarrow B \in \mathcal{T}$ iff $\neg A \in \mathcal{T}$ or $B \in \mathcal{T}$; ELt3logics: $A \rightarrow B \in \mathcal{T}$ iff $A \notin \mathcal{T}$ or $B \in \mathcal{T} ;$ ELt4-logics: $A \rightarrow B \in \mathcal{T}$ iff $\neg A \in \mathcal{T}$ or $\neg B \notin \mathcal{T}$; ELt5-logics: $A \rightarrow B \in \mathcal{T}$ iff $\neg A \in \mathcal{T}$ or $B \in \mathcal{T}$; ELt6-logics: $A \rightarrow B \in \mathcal{T}$ iff $\neg A \in \mathcal{T}$ or $\neg B \notin \mathcal{T}$. 
Proof. It is similar to those of Propositions 5.5 through 5.8. (I) ELt1-logics $(\Rightarrow)$ Suppose (1) $A \rightarrow B \in \mathcal{T}$ and, for reductio, (2) $A \in \mathcal{T} \quad \& \neg B \in \mathcal{T} \quad \& \neg A \notin \mathcal{T}$ or (3) $A \in \mathcal{T} \& \neg B \in \mathcal{T} \& B \notin \mathcal{T}$. But (2) and (3) are impossible by $[(A \rightarrow$ $B) \wedge \neg B] \rightarrow \neg A($ A15) and $[(A \rightarrow B) \wedge A] \rightarrow B$ (A14), respectively. $(\Leftarrow)(1)$ Suppose $A \notin \mathcal{T}$. By completeness, we have $\neg A \in \mathcal{T}$. Then, $A \rightarrow B \in \mathcal{T}$ follows by $\neg A \rightarrow[A \vee(A \rightarrow B)]$ (A9) and primeness. (2) Suppose $\neg B \notin \mathcal{T}$. We deduce $A \rightarrow B \in \mathcal{T}$ similarly as in case (1) but by using now $B \rightarrow[\neg B \vee(A \rightarrow B)]$ (A10). (3) Suppose $\neg A \in \mathcal{T}$ and $B \in \mathcal{T}$. Further, let $C$ be an arbitrary wff and, for reductio, $A \rightarrow B \notin \mathcal{T}$. By completeness, we obtain $\neg(A \rightarrow B) \in \mathcal{T}$, whence, by $[\neg(A \rightarrow B) \wedge(\neg A \wedge B)] \rightarrow C$ (A11), $C \in \mathcal{T}$ is derivable, contradicting the a-consistency of $\mathcal{T}$.

The cases for ELt2-logics, ELt3-logics, ELt4-logics, ELt5-logics and ELt6logics are proved similarly. In particular, it suffices to use the properties of $\mathcal{T}$ mentioned in the statement of Proposition 5.11 and the following theorems. ELt2-logics: $[(A \rightarrow B) \wedge A] \rightarrow(\neg A \vee B)(\mathrm{A} 12), A \rightarrow(B \rightarrow A)(\mathrm{A} 16)$ and $\neg A \rightarrow$ $(A \rightarrow B)$ (A17). ELt3-logics: $\neg A \rightarrow[A \vee(A \rightarrow B)]($ A9), $[(A \rightarrow B) \wedge A] \rightarrow B$ (A14) and A16. ELt4-logics: $B \rightarrow[\neg B \vee(A \rightarrow B)]$ (A10), $[(A \rightarrow B) \wedge \neg B] \rightarrow \neg A$ (A15) and A17. ELt5-logics: A14, A16 and A17. ELt6-logics: A16, A17 and $[(A \rightarrow B) \wedge(A \wedge \neg B)] \rightarrow C(\mathrm{~A} 25)$.

Proposition $5.12(\rightarrow$ in prime, regular, consistent Lt $i$-theories) Let $L$ be an ELti-logic where Lti will refer in each case to one of the extensions of $b^{3}$ displayed in Definitions 3.7 and 3.10. And let $\mathcal{T}$ be a prime, regular and consistent L-theory. We have: ELt1-logics: $\neg(A \rightarrow B) \in \mathcal{T}$ iff $[A \in \mathcal{T}$ and $B \notin \mathcal{T}]$ or $[\neg A \notin \mathcal{T}$ and $\neg B \in \mathcal{T}] ;$ ELt2-logics: $\neg(A \rightarrow B) \in \mathcal{T}$ iff $A \in \mathcal{T}$ and $\neg B \in \mathcal{T}$; ELt3-logics: $\neg(A \rightarrow B) \in \mathcal{T}$ iff $\neg A \notin \mathcal{T}$ and $\neg B \in \mathcal{T}$; ELt4-logics: $\neg(A \rightarrow B) \in \mathcal{T}$ iff $A \in \mathcal{T}$ and $B \notin \mathcal{T} ;$ ELt5-logics: $\neg(A \rightarrow B) \in \mathcal{T}$ iff $A \in \mathcal{T}$ and $\neg B \in \mathcal{T}$; ELt6-logics: $\neg(A \rightarrow B) \in \mathcal{T}$ iff $A \in \mathcal{T}$ and $B \notin \mathcal{T}$.

Proof. Similar to those of Propositions 5.5 through 5.8. In particular, it suffices to use the primeness, regularity and consistency of $\mathcal{T}$ together with the following axioms and theorems.

ELt1-logics: $\neg A \rightarrow[A \vee(A \rightarrow B)]($ A9),$B \rightarrow[\neg B \vee(A \rightarrow B)]$ (A10), $[\neg(A \rightarrow B) \wedge(\neg A \wedge B)] \rightarrow C(\mathrm{~A} 11),(A \vee \neg B) \vee(A \rightarrow B)(\mathrm{T} 6), A \rightarrow[B \vee \neg(A \rightarrow$ $B)]$ (T9) and $\neg B \rightarrow[\neg A \vee \neg(A \rightarrow B)]$ (T10).

(T11).

ELt2-logics: $(A \wedge \neg B) \rightarrow[\neg(A \rightarrow B) \vee \neg A](\mathrm{T} 7)$ and $\neg(A \rightarrow B) \rightarrow(A \wedge \neg B)$

ELt3-logics: $[\neg(A \rightarrow B) \wedge \neg A] \rightarrow A$ (T4), $\neg B \rightarrow[\neg A \vee \neg(A \rightarrow B)]$ (T10) and $\neg(A \rightarrow B) \rightarrow \neg B$ (T12).

ELt4-logics: $A \rightarrow[B \vee \neg(A \rightarrow B)](\mathrm{T} 9),[\neg(A \rightarrow B) \wedge B] \rightarrow \neg B$ (T5) and $\neg(A \rightarrow B) \rightarrow A(\mathrm{~T} 13)$.

ELt5-logics: $\neg(A \rightarrow B) \leftrightarrow(A \wedge \neg B)($ A23).

ELt6-logics: $A \rightarrow(B \rightarrow A)$ (A16), $A \vee(A \rightarrow B)(\mathrm{A} 18)$ and $A \rightarrow[B \vee \neg(A \rightarrow$ $B)]$ (A24).

Let us develop, for example, the case of ELt3-logics. $(\Rightarrow)$ Suppose $\neg(A \rightarrow$ $B) \in \mathcal{T}$. If $\neg A \in \mathcal{T}$, then $A \in \mathcal{T}$ follows by $[\neg(A \rightarrow B) \wedge \neg A] \rightarrow A$ (T4) 
contradicting the consistency of $\mathcal{T}$. Thus, $\neg A \notin \mathcal{T}$. On the other hand, $\neg B \in \mathcal{T}$ is immediate by $\neg(A \rightarrow B) \rightarrow \neg B(\mathrm{~T} 12) . \quad(\Leftarrow)$ Suppose $\neg A \notin \mathcal{T}$ and $\neg B \in \mathcal{T}$. Then $\neg(A \rightarrow B) \in \mathcal{T}$ is deducible by the primeness of $\mathcal{T}$ and $\neg B \rightarrow[\neg A \vee \neg(A \rightarrow$ $B)](\mathrm{T} 10)$.

\section{Completeness of the Lt $i$-logics}

We prove the (weak) completeness of the Lt $i$-logics $(1 \leq i \leq 6)$ by using canonical model constructions. As we have defined two types of models, uLt $i$-models and oLt $i$-models (cf. Definitions 4.2 and 4.5), we need two types of canonical models, "canonical uLt $i$-models" and "canonical oLt $i$-models", which are based upon the more basic notions of $\mathcal{T}_{\mathrm{Lt} i}^{\mathrm{u}}$-interpretations and $\mathcal{T}_{\mathrm{Lt} i}^{\mathrm{o}}$-interpretations, respectively. We begin by defining the latter notions.

Definition 6.1 ( $\mathcal{T}_{\mathrm{Lt} i}^{\mathbf{u}}$-interpretations) Let $K$ be the set $\{\{T\},\{F\}, \emptyset\}$ as in Definition 4.1. And let $\mathcal{T}$ be a prime, regular and consistent Lti-theory. Then, the function $I$ from the set of all wffs to $K$ is defined as follows: for each propositional variable $p$, we set (a) $T \in I(p)$ iff $p \in \mathcal{T}$; (b) $F \in I(p)$ iff $\neg p \in \mathcal{T}$. Next, $I$ assigns a member of $K$ to each wff $A$ according to clauses $2 a, 2 b$, 3a, 3b, 4a, 4b, 5a1 (or 5a2) and $5 b i(1 \leq i \leq 6)$ in Definition 4.2. Then, it is said that $I$ is a $\mathcal{T}_{\text {Lti }}^{u}$-interpretation.

Definition $6.2\left(\mathcal{T}_{\mathbf{L t} i}^{\mathbf{o}}\right.$-interpretations) Let $K$ be the set $\{\{T\},\{F\},\{T, F\}\}$ as in Definition 4.4. And let $\mathcal{T}$ be a prime, complete and a-consistent Ltitheory. Then, the function I from the set of all wffs to $K$ is defined similarly as in Definition 6.1 in the case of propositional variables, but complex wffs are assigned a member of $K$ according to clauses 2a, 2b, 3a, 3b, 4a, 4b, 5ai $(1 \leq i \leq 6)$ and $5 b 1$ (or 5b2) in Definition 4.5. Then, it is said that $I$ is a $\mathcal{T}_{\text {Lti }}^{o}$-interpretation.

Now, we can defined the canonical models.

Definition 6.3 (Canonical uLt $i$-models) A canonical uLti-model is the structure $\left(K, I_{\mathcal{T} u}\right)$ where $K$ is defined as in Definition 4.1 and $I_{\mathcal{T} u}$ is a $\mathcal{T}_{\text {Lti }}^{u}$-interpretation defined upon the Lti-theory $\mathcal{T}$.

Definition 6.4 (The canonical relation $\vDash_{I_{\mathcal{T} u}}$ ) Let $\left(K, I_{\mathcal{T} u}\right)$ be a canonical uLti-model. Then for any formula $A, \vDash_{I_{\mathcal{T}} u} A$ ( $A$ is a true in the canonical uLti-model $\left.\left(K, I_{\mathcal{T} u}\right)\right)$ iff $T \in I_{\mathcal{T} u}(A)$.

Definition 6.5 (Canonical oLt $i$-models) A canonical oLti-model is the structure $\left(K, I_{\mathcal{T}_{o}}\right)$ where $K$ is defined as in Definition 4.4 and $I_{\mathcal{T}_{o}}$ is a $\mathcal{T}_{\text {Lti }}^{o}$-interpretation defined upon the Lti-theory $\mathcal{T}$.

Definition 6.6 (The canonical relation $\vDash_{I_{\mathcal{T}_{\mathrm{o}}}}$ ) Let $\left(K, I_{\mathcal{T}_{o}}\right)$ be a canonical oLti-model. Then for any formula $A, \vDash_{I_{\mathcal{T}} \text { o }} A$ ( $A$ is a true in the canonical oLti-model $\left.\left(K, I_{\mathcal{T} o}\right)\right)$ iff $F \notin I_{\mathcal{T} o}(A)$. 
We immediately have the following proposition.

Proposition 6.7 (Canonical Lt $i$-models are indeed Lt $i$-models) Let $M$ be a canonical uLti-model (resp. oLti-model). Then, $M$ is indeed a uLti-model (resp., oLti-model).

Proof. Immediate by Definitions 4.2 and 6.3 (respectively, 4.5 and 6.5).

Given Proposition 6.7, the essential fact we have to prove in order to prove completeness is that conditions (a) and (b) for propositional variables in Definitions 6.1 and 6.2 can be generalized to all formulas. This fact is proved in Lemmas 6.8 and 6.9 below.

Lemma 6.8 $\left(\mathcal{T}_{\mathrm{Lt} i}^{\mathrm{u}}\right.$-interpreting the set of formulas) Let I be a $\mathcal{T}_{L t i}^{u}$-interpretation defined upon the Lti-theory $\mathcal{T}$. For each wff $A$, we have: (1) $T \in I(A)$ iff $A \in \mathcal{T}$; (2) $F \in I(A)$ iff $\neg A \in \mathcal{T}$.

Lemma $6.9\left(\mathcal{T}_{\mathbf{L t} i}^{\mathrm{o}}\right.$-interpreting the set of formulas) Let I be a $\mathcal{T}_{L t i}^{o}$-interpretation defined upon the Lti-theory $\mathcal{T}$. For each wff $A$, we have: (1) $T \in I(A)$ iff $A \in \mathcal{T}$; (2) $F \in I(A)$ iff $\neg A \in \mathcal{T}$.

The proof of Lemma 6.8 (resp., Lemma 6.9) is by induction on the length of $A$ and is based upon the properties of prime, regular and consistent Lt $i$-theories (resp., prime, complete, and a-consistent Lt $i$-theories) recorded in Propositions 5.3, 5.4, 5.6 (resp., 5.5), 5.8 (resp., 5.7) and 5.12 (resp., 5.11). By using the aforementioned propositions, it is essentially shown that the clauses in Definition 4.2 (resp., Definition 4.5) hold in the corresponding uLt $i$-models (resp., oLtimodels). The proof is similar to that of Lemma 9.5 in [19] (resp., Lemma 8.5 in [17]). In the following proof, we provide details for two of the cases.

Proof. Lt1. ULt1-interpretations. $F \in(A \rightarrow B)$ iff (clause 5b1 -Definition 4.2) $[T \in I(A)$ and $T \notin I(B)]$ or $[F \notin I(A)$ and $F \in I(B)]$ iff (induction) $[A \in \mathcal{T}$ and $B \notin \mathcal{T}]$ or $[\neg A \notin \mathcal{T}$ and $\neg B \in \mathcal{T}]$ iff (Proposition 5.12) $\neg(A \rightarrow B) \in \mathcal{T}$.

Lt5. OLt5-interpretations. $T \in I(A \rightarrow B)$ iff (clause $5 \mathrm{a} 2$-Definition 4.5) $F \in I(A)$ or $T \in I(B)$ iff (induction) $\neg A \in \mathcal{T}$ or $B \in \mathcal{T}$ iff (Proposition 5.11) $A \rightarrow B \in \mathcal{T}$.

Now, we can prove weak completeness of the Lti-logics w.r.t. the respective matrix Mti.

Theorem 6.10 (Completeness of Lt $i$-logics w.r.t. $\vDash_{\mathrm{Mt} i}$ ) For any wff $A$, if $\vDash_{M t i} A$, then $\vdash_{L t i} A$.

Proof. Suppose that $A$ is a wff such that $\nvdash_{\mathrm{Lt} i} A$. We prove $\nvdash_{\mathrm{Mt} i} A$. Let Lt $i$ be the set of its theorems. By the Primeness Lemma (Lemma 5.10), there is a prime Lti-theory $\mathcal{T}$ such that Lt $i \subseteq \mathcal{T}$ and $A \notin \mathcal{T}$, whence it follows that $\mathcal{T}$ is, in addition, regular and a-consistent. Suppose that $\mathcal{T}$ is inconsistent. Then, $\mathcal{T}$ is complete (by Proposition 5.9) and so $\neg A \in \mathcal{T}$. Moreover, $\mathcal{T}$ generates a canonical oLt $i$-model $\left(K, I_{\mathcal{T}}\right.$ o defined upon the Lt $i$-theory $\mathcal{T}$ (cf. Definition $6.5)$ such that $F \in I_{\mathcal{T}_{\mathrm{o}}}(A)$, i.e., $\nvdash_{I_{\mathcal{T}_{\mathrm{o}}}} A$, and consequently, $\nvdash_{\mathrm{Lt} i}^{\mathrm{o}} A$ by Definition 
6.6. But, on the other hand, suppose that $\mathcal{T}$ is consistent. Then $\mathcal{T}$ generates a canonical uLt $i$-model $\left(K, I_{\mathcal{T}}\right.$ u $)$ defined upon the Lt $i$-theory $\mathcal{T}$ (cf. Definition 6.3) such that $T \notin I_{\mathcal{T} \mathrm{u}}(A)$, i.e., $\nvdash_{I \mathrm{u}} A$, and therefore, $\nvdash_{\mathrm{Lt} i}^{\mathrm{u}} A$ by Definition 6.4. In consequence, if $\nvdash_{\mathrm{Lt} i} A$ either $\nvdash_{\mathrm{Lt} i}^{\mathrm{o}} A$ or else $\nvdash_{\mathrm{Lt} i}^{\mathrm{u}} A$. So, by Proposition 4.10, $\nvdash_{\mathrm{Mt} i} A$ follows, as it was to be proved.

As a corollary of Theorem 6.10 , we have completeness of Lt $i$ w.r.t. $\models_{\mathrm{Lt} i}^{\mathrm{o}}$ and $\vDash_{\mathrm{Lt} i}^{\mathrm{u}}$.

Corollary 6.11 (Completeness of Lt $i$ w.r.t. $\vDash_{\mathbf{L} t i}^{\mathbf{o}}$ and $\models_{\mathbf{L} \mathbf{t} i}^{\mathbf{u}}$ ) For any wff $A$, (1) if $\vDash_{L t i}^{o} A$, then $\vdash_{L t i} A$; and (2) if $\vDash_{L t i}^{u} A$, then $\vdash_{L t i} A$.

Proof. Immediate by Theorem 6.10 and Proposition 4.10.

\section{The logics Lt $i^{2}$ and $\mathbf{L t} i \leq$}

So far, logics have been understood as sets of theorems or, equivalently, as sets of valid formulas. In this section, logics will be viewed in a more general way as structures determined by consequence relations. We begin by defining the notions of truth-preserving and degree of truth-preserving consequence relations in the context of the natural implicative expansions of MK3 with only one designated value.

Definition 7.1 (The relations $\vDash_{\mathrm{Mt} i}^{2}$ and $\vDash_{\mathrm{M} t i}^{\leq}$) Let $M t i(1 \leq i \leq 6)$ be one of the natural implicative expansions of MK3 defined in Definition 2.9(b). There are essentially two different ways of defining a consequence relation in Mti: truth-preserving relation (denoted by $\vDash_{M t i}^{2}$ ) and degree of truth preserving relation (denoted by $\vDash \leq_{M}$ ). These relations are defined as follows for any set of wffs $\Gamma$ and wff $A$ : (1) $\Gamma \vDash_{M t i}^{2} A$ iff $I(A) \in D$ whenever $I(\Gamma) \in D$ for all Mti-interpretations I; (2) $\Gamma \vDash \frac{\leq}{M} t i$ iff $a \leq I(A)$ whenever $a \leq I(\Gamma)$ for all $a \in \mathcal{V}$ and Mti-interpretations $I .[T \in I(\Gamma)$ iff $\forall A \in \Gamma(T \in I(A)) ; F \in I(\Gamma)$ iff $\exists A \in \Gamma(F \in I(A)) ;[I(\Gamma)=\inf \{I(B) \mid B \in \Gamma\}]$.

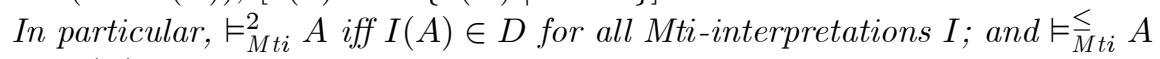
iff $a \leq I(A)$ for all $a \in \mathcal{V}$ and for all Mti-interpretations $I$.

$\Gamma \models_{M t i}^{2} A$ (resp., $\Gamma \models_{\bar{M} t i}^{\leq} A$ ) can be read " $A$ is a consequence of $\Gamma$, according to $M$ ti in the truth-preserving (resp., degree of truth-preserving) sense". And $\vDash_{M t i}^{2} A$ (resp., $\vDash_{M t i}^{\leq} A$ ) can be read " $A$ is Mti-valid or $A$ is valid in the matrix Mti in the truth-preserving (resp., degree of truth-preserving) sense".

These two notions of consequence are not equivalent as it is shown below.

Proposition 7.2 (Relation between $\vDash_{\mathrm{Mt} i}^{2}$ and $\left.\vDash_{\mathrm{M} \mathrm{t} i}^{\leq}\right)$Let $M t i(1 \leq i \leq 6)$ be one of the natural implicative expansions of $M K 3$ defined in Definition 2.9(b). For any set of wffs $\Gamma$ and any wff $A$, (1) if $\Gamma \vDash_{M}^{\leq} A$, then $\Gamma \vDash_{M t i}^{2} A$; (2) the converse of (1), however, does not hold. 
Proof. (1) It is immediate. (2) $\{A, \neg A\} \vDash_{\mathrm{Mt} i}^{2} B$ holds for any wffs $A, B$. Consider, however, different propositional variables $p_{i}, p_{j}$ and Mt $i$-interpretation $I$ such that $I\left(p_{i}\right)=1$ and $I\left(p_{j}\right)=0$. Then, $\inf \left\{p_{i}, \neg p_{i}\right\}=1$, given this Mtiinterpretation $I$.

However, we note the following remark.

Remark $7.3\left(\models_{\overline{\mathrm{M}} \mathrm{t} i}^{\leq} A\right.$ iff $\left.\models_{\mathrm{Mt} i}^{2} A\right)$ Let Mti $(1 \leq i \leq 6)$ be one of the natural implicative expansions of MK3 defined in Definition 2.9(b). Notice that $\models_{M}^{\leq} A$ iff $I(A)=2$ for all $\mathcal{M}$ ti-interpretations $I$. Thus, for every wff $A, \vDash \stackrel{\leq}{M} t i$ iff $\vDash_{M t i}^{2} A$.

Consider now the following definition.

Definition 7.4 (Logics determined by matrices) Let $\mathcal{L}$ be a propositional language, $M$ a matrix for $\mathcal{L}$ and $\vdash_{L}$ a (proof theoretical) consequence relation defined on $\mathcal{L}$. Then, the logic $L$ (cf. Definition 2.2) is determined by $M$ iff for every set of wffs $\Gamma$ and wff $A, \Gamma \vdash_{L} A$ iff $\Gamma \vDash_{M} A$. In particular, the logic $L$ (considered as the set of its theorems) is determined by M iff for every wff $A$, $\vdash_{L} A$ iff $\vDash_{M} A$ (cf. Definition 2.5). Notice that $\vDash_{M}$ can be understood in the truth-preserving or degree of truth-preserving sense.

Therefore, given the (weak) soundness and completeness theorems (Theorems 4.11 and 6.10), we have that Lti, understood as the set of all theorems of Lt $i$, is the logic determined by the matrix Mt $i$ in the sense of Definition 7.4. That is, the set of all valid formulas according to Mti and the set of all theorems of Lt $i$ are one and the same set $(1 \leq i \leq 6)$.

Now, let us name Lt $i^{2}$ (resp., Lt $i \leq$ ) the logic determined by the relation $\vDash_{\mathrm{Mt} i}^{2}$ (resp., $\left.\vDash_{\mathrm{M} t i}^{\leq}\right)$. The aim of this section is to define, for all $i(1 \leq i \leq 6)$, the logics $\mathrm{Lt} i^{2}$ and Lt $i \leq$ determined by the relations $\vDash_{\mathrm{Mt} i}^{2}$ and $\models_{\mathrm{M} t i}^{\leq}$, respectively. In order to fulfill this aim, we define relations equivalent to $\vDash_{\mathrm{Mt} i}^{2}$ and $\vDash_{\mathrm{M} t i}^{\leq}$ in uLti-semantics and o-Lti-semantics. Then, we investigate which deductive consequence relations $\models_{\mathrm{Mt} i}^{2}$ and $\models_{\mathrm{M} t i}^{\leq}$are equivalent to.

Definition 7.5 (The relations $\models_{\mathbf{L t} i}^{\mathbf{u} 2}$ and $\left.\models_{\mathbf{L t} i}^{\mathbf{o} 2}\right)$ For any $i(1 \leq i \leq 6)$, set of wffs $\Gamma$ and wff $A$, (1) $\Gamma \vDash_{L t i}^{u 2} A$ iff $T \in I(A)$ whenever $T \in I(\Gamma)$ for all uLti-interpretations I; (2) $\Gamma \models_{L t i}^{\text {o2 }} A$ iff $F \notin I(A)$ whenever $F \notin I(\Gamma)$ for all oLti-interpretations $I$.

Definition 7.6 (The relations $\models_{\mathbf{L} t i}^{\mathbf{u}} \leq$ and $\models_{\mathbf{L} t i}^{\mathbf{o}} \leq$ ) For any set of wffs $\Gamma$ and wff $A$ : (1) $\Gamma \vDash_{L t i}^{u \leq} A$ iff $F \in I(\Gamma)$ or $T \in I(A)$ or $(T \notin I(\Gamma)$ and $F \notin I(A)$ ) for all uLti-interpretations $I$. (2) $\Gamma \vDash_{L t i}^{o \leq} A$ iff $T \in I(A)$ whenever $T \in I(\Gamma)$ and $F \notin I(A)$ whenever $F \notin I(\Gamma)$ for all oLti-interpretations $I$.

Before proving the coextensiveness of the relations $\models_{\mathrm{Mt} i}^{2}, \models_{\mathrm{Lt} i}^{\mathrm{u} 2}$ and $\models_{\mathrm{Lt} i}^{\mathrm{o} 2}$ (resp., $\vDash_{\overline{\mathrm{M}} t i}^{\leq}, \models_{\mathrm{Lt} i}^{\mathrm{u} \leq}$ and $\vDash_{\mathrm{Lt} i}^{\mathrm{O}} \leq \mathrm{C}$, we note the following corollary of Proposition 4.9. 
Proposition 7.7 (Equivalence of corresponding interpretations II) (I) Let I be a u-interpretation (resp., Mti-interpretation) and $I^{\prime}$ the Mti-interpretation (resp., u-interpretation) corresponding to $I$. Then, for each set of $w f f s ~ \Gamma$, we have: (1) $I(\Gamma)=\{T\}$ iff $I^{\prime}(\Gamma)=2 ; I(\Gamma)=\emptyset$ iff $I^{\prime}(\Gamma)=1$; and (3) $I(\Gamma)=\{F\}$ iff $I^{\prime}(\Gamma)=0$.

(II) Let I be an o-interpretation (resp., Mti-interpretation) and I' the Mtiinterpretation (resp., o-interpretation) corresponding to $I$. Then, for each set of wffs $\Gamma$, we have: (1) $T \in I(\Gamma)$ iff $I^{\prime}(\Gamma)=2$ or $I^{\prime}(\Gamma)=1$; (2) $F \in I(\Gamma)$ iff $I^{\prime}(\Gamma)=0$ or $I^{\prime}(\Gamma)=1$.

Proof. Immediate by Proposition 4.9.

We prove:

Proposition 7.8 (Coextensiveness of $\vDash_{\overline{\mathrm{M}} \mathrm{t} i}^{\leq}, \vDash_{\mathrm{Mt} i}^{\mathbf{u} \leq}$ and $\models_{\mathrm{Mt} i}^{\mathbf{o}} \leq$ ) For any set of wffs $\Gamma$ and any wff $A$, (1) $\Gamma \vDash_{M t i}^{\leq} A$ iff $\Gamma \vDash_{M t i}^{u \leq} A$; (2) $\Gamma \vDash_{M t i}^{\leq} A$ iff $\Gamma \vDash_{M t i}^{o \leq} A$.

Proof. Firstly, we prove $(2)(\Rightarrow)$ Suppose $\Gamma \vDash_{\overline{\mathrm{M}} \mathrm{t} i}^{\leq} A$ and let $I$ be an arbitrary oLti-interpretation. We have to prove $T \in I(\Gamma) \Rightarrow T \in I(A)$ and $F \notin I(\Gamma) \Rightarrow$ $F \notin I(A)$. We define the Mti-interpretation $I^{\prime}$ corresponding to $I$ (cf. Definition 4.8). Then, we have: (a) Suppose $T \in I(\Gamma)$. We consider two subcases: $F \in I(\Gamma)$ and $F \notin I(\Gamma)$. If $F \in I(\Gamma)$, then $I^{\prime}(\Gamma)=1$ (Proposition 7.7) and, by the hypothesis, $I^{\prime}(A)=1$ or $I^{\prime}(A)=2$, that is, $T \in I(A)$ (Proposition 4.9). If $F \notin I(\Gamma)$, then $I^{\prime}(\Gamma)=2$ (Proposition 7.7), and by the hypothesis, $I^{\prime}(A)=2$, that is, $T \in I(A)$ (and $F \notin I(A)$ ), by Proposition 4.9. (b) Suppose $F \notin I(\Gamma)$. Then, $T \in I(\Gamma)$ since $T \notin I(\Gamma)$ is not possible, $I$ being an o-interpretation. So, case (b) is proved as the second subcase of (a).

$(2)(\Leftarrow)$ We omit references to Definition 4.8 and Propositions 4.9 and 7.7. Suppose $\Gamma \models_{\mathrm{Lt} i}^{\mathrm{o}} A$ and let $I$ be an arbitrary Mti-interpretation. We define the oLti-interpretation $I^{\prime}$ corresponding to $I$. Next, we consider the three possible values that $I$ can assign to $\Gamma$. (a) $I(\Gamma)=0$. Then, $I(\Gamma) \leq I(A)$ is immediate. (b) $I(\Gamma)=1$. Then, $T \in I^{\prime}(\Gamma)$ and $F \in I^{\prime}(\Gamma)$. By the hypothesis, $T \in I^{\prime}(A)$. Now, if $F \in I^{\prime}(A)$, then $I(A)=1$; and if $F \notin I^{\prime}(A)$ then $I(A)=2$. Thus, in either case, we have $I(\Gamma) \leq I(A)$. (c) $I(\Gamma)=2$. Then, $T \in I^{\prime}(\Gamma)$ and $F \notin I^{\prime}(\Gamma)$. By the hypothesis, $T \in I^{\prime}(A)$ and $F \notin I^{\prime}(A)$, whence $I(A)=2$. So, $I(\Gamma) \leq I(A)$.

(1) The proof is similar (cf. Proposition 12 in [18]).

If $\Gamma$ is the empty set, then the proof follows by Proposition 4.9.

Proposition 7.9 (Coextensiveness of $\models_{\mathbf{M t} i}^{2}, \models_{\mathbf{L t} i}^{\mathbf{u} 2}$ and $\left.\models_{\mathbf{L t} i}^{\mathbf{o} 2}\right)$ For any $i(1 \leq$ $i \leq 6$ ), set of wffs $\Gamma$ and wff $A$, (1) $\Gamma \vDash_{M t i}^{2} A$ iff $\Gamma \vDash_{L t i}^{u 2} A$; (2) $\Gamma \vDash_{M t i}^{2} A$ iff $\Gamma \models_{L t i}^{o 2} A$.

Proof. Immediate given Proposition 7.7 and Definitions 7.1 and 7.5.

In what follows, we investigate which deductive consequence relations the relations $\models_{\mathrm{Mt} i}^{2}$ and $\models_{\mathrm{M} t i}^{<}$correspond to.

Let $\mathrm{L}$ be an Lti-logic. The standard deductive consequence relation can be defined as follows. 
Definition 7.10 (Standard deductive consequence relation) Let $L$ be an Lti-logic, $\Gamma$ be a set of wffs and $A$ a wff. Then, $\Gamma \vdash_{L}^{2} A$ ( $A$ is derivable from $\Gamma$ in L) iff there is a finite sequence of wffs $B_{1}, \ldots, B_{m}$ such that $B_{m}$ is $A$ and for each $k(1 \leq k \leq m)$ one of the following is the case: (1) $B_{k} \in \Gamma$; (2) $B_{k}$ is an axiom of $L ;$ (3) $B_{k}$ is the result of applying one of the rules Adj, MP, Suf, Pref, Veq and Con (when present: in Lt1-Lt4) to one or two of the preceding wffs in the sequence (cf. Definitions 3.1, 3.4, 3.7 and 3.10). By $\vdash_{L}^{2}$, we shall refer to this deductive relation and, by $L t i^{2}$, to the logic $\left(\mathcal{L}, \vdash_{L t i}^{2}\right)$ (cf. Definition 2.2).

In [19] (Theorems 8.6 and 9.8), the following theorem is proved.

Theorem 7.11 (Strong sound. and compl. of Lt $i^{2}$ w.r.t. $\vDash_{\text {Mt } i}^{2}$ ) For any $i(1 \leq i \leq 6)$, set of wffs $\Gamma$ and $w f f A, \Gamma \vdash_{L t i}^{2} A$ iff $\Gamma \vDash_{M t i}^{2} A$.

Consequently, given Proposition 7.9, we have the following corollary.

Corollary 7.12 (Strong sound. and compl. of Lt $i^{2}$ w.r.t. $\models_{\mathbf{L t} i}^{\mathbf{u} 2} \& \vDash_{\mathbf{L t} i}^{\mathbf{o} 2}$ ) For any $i(1 \leq i \leq 6)$, set of wffs $\Gamma$ and wff $A$, (1) $\Gamma \vdash_{L t i}^{2} A$ iff $\Gamma \vDash_{L t i}^{u 2} A$; (2) $\Gamma \vdash_{L t i}^{2} A$ iff $\Gamma \models_{L t i}^{o 2} A$.

Proof. Immediate by Proposition 7.9 and Theorem 7.11.

As we have seen, the deductive relation just defined corresponds to the truthpreserving relation $\models_{\mathrm{Mt} i}^{2}$. We consider an alternative to this standard deductive relation.

Definition 7.13 (Alternative deductive consequence relation) Let $L$ be an Lti-logic, $\Gamma$ be a set of wffs and $A$ a wff. Then, $\Gamma \vdash_{\bar{L}}^{\leq} A$ ( $A$ is derivable from $\Gamma$ in $L)$ iff there is a finite sequence of wffs $B_{1}, \ldots, B_{m}$ such that $B_{m}$ is $A$ and for each $k(1 \leq k \leq m)$ one of the following is the case: (1) $B_{k} \in \Gamma$; (2) $B_{k}$ is a theorem of $L$; (3) $B_{k}$ is the result of applying Adj or L-entailment to two previous formulas in the sequence. By $\vdash_{L}$, we shall refer to this deductive relation and, by $L t i \leq$, to the logic $(\mathcal{L}, \vdash \stackrel{\leftarrow}{L} t i)$ (cf. Definition 2.2).

We will prove $\Gamma \vdash_{\mathrm{Lt} i}^{\leq} A$ iff $\Gamma \vDash_{\mathrm{M} t i}^{\leq} A$ for each $i(1 \leq i \leq 4)$. We begin by proving soundness. As pointed out below Lt $5 \leq$ (resp., Lt $6 \leq$ ) is not sound w.r.t. $\vDash_{\overline{\mathrm{M}} \mathrm{t} 5}^{\leq}\left(\right.$resp.,,$\left.\vDash_{\mathrm{M} \mathrm{t} 6}^{\leq}\right)$.

Theorem 7.14 (Soundness of Lt $i$ w.r.t. $\left.\vDash_{\mathrm{M} t i}^{\leq}\right)$For any $i(1 \leq i \leq 4)$, set of wffs $\Gamma$ and wff $A$, if $\Gamma \vdash \stackrel{\leq t i}{\leq} A$, then $\Gamma \vDash \stackrel{\leq}{M} t i$.

Proof. The proof is by induction on the length of the proof of $A$ from $\Gamma$. If $A \in \Gamma$ or $A$ is by Adj, the proof is trivial; and it is immediate if $A$ is a theorem of Lt $i$, since, by Theorem 4.11, all theorems of Lt $i$ are Mti-valid. So, suppose that $A$ is by L-entailment. Then, $\Gamma \vdash_{\mathrm{Lt} i}^{\leq} B$ and $\vdash_{\mathrm{Lt} i} B \rightarrow A$ for some wff $B$. Let now $I$ be an arbitrary Mti-interpretation. We have to show $I(\Gamma) \leq I(A)$. By hypothesis, we have $I(\Gamma) \leq I(B)$; and, by Theorem 4.11, $I(B \rightarrow A)=2$. Then, there are three possibilities to consider. 
1. $I(B)=2$. Then, $I(A)=2$, according to Mti (cf. Definition 2.9(b)). So, $I(\Gamma) \leq I(A)$.

2. $I(B)=0$. Then, clearly $I(\Gamma) \leq I(A)$.

3. $I(B)=1$. Then, $I(A)=1$ or $I(A)=2$, according to Mti (cf. Definition $2.9(\mathrm{~b}))$. So, $I(\Gamma) \leq I(A)$.

Remark 7.15 (Lt5 (resp., Lt6) is not sound w.r.t. $\vDash_{\overline{\mathrm{M}} \mathrm{t} 5}^{<}$(resp., $\left.\vDash_{\overline{\mathrm{M}} \mathrm{t} 6}^{<}\right)$) Notice that Lt5-ent (resp., Lt6-ent) is not verified by $\models_{\bar{M}+5}^{\leq}$-semantics (resp., $\vDash \frac{\leq}{M} 6^{-}$-semantics). Consider any Mt5-interpretation I (resp., Mt6-interpretation I) such that $I(B)=1$ and $I(A)=0$. Then, $I(B \rightarrow A)=2$, but $I(\Gamma)>I(A)$.

As a corollary, we have soundness w.r.t. $\models_{\mathrm{Mt} i}^{\mathrm{o}} \leq$ and $\vDash_{\mathrm{Mt} i}^{\mathrm{u}}$.

Corollary 7.16 (Soundness of Lt $i$ w.r.t. $\vDash_{\mathbf{L} \mathbf{t} i}^{\mathbf{o}} \leq$ and $\left.\models_{\mathbf{L} t i}^{\mathbf{u}} \leq \mathbf{i}\right)$ For any $i(1 \leq$ $i \leq 4)$, set of wffs $\Gamma$ and wff $A$, if $\Gamma \vdash \stackrel{L}{\leq} A$, then (1) $\Gamma \vDash_{L t i}^{o \leq} A$ and (2) $\Gamma \vDash_{L t i}^{u \leq} A$.

Proof. Immediate by Proposition 7.8 and Theorem 7.14.

Contrary to what the case with soundness has been, in what follows we prove completeness of Lt $i \leq$ for all $i(1 \leq i \leq 6)$ w.r.t. $\vDash_{\mathrm{M} t i}^{\leq}$and, consequently, w.r.t. $\models_{\mathrm{Lt} i}^{\mathrm{o}} \leq$ and $\models_{\mathrm{Lt} i}^{\mathrm{u}} \leq$.

Firstly, we recall the notion of the set of consequences of a given set of formulas in Lt $i$ and then we prove completeness.

Definition 7.17 (The set of consequences of $\Gamma$ in Lt $i$ ) The set of consequences in Lti of a set of wffs $\Gamma$ (in symbols, $C n \Gamma[L t i]$ ) is defined as follows: $C n \Gamma[L t i]=\left\{A \mid \Gamma \vdash_{L t i}^{\leq} A\right\}$.

We note the following remark.

Remark 7.18 (The set of consequences of $\Gamma$ in Lt $i$ is a regular theory) It is obvious that for any $\Gamma, C n \Gamma[L t i]$ is a regular theory: it is closed by Adj, Lti-ent, and contains all theorems of Lti (cf. Definitions 5.2 and 7.13).

Theorem 7.19 (Completeness w.r.t. $\left.\vDash \frac{\leq}{\mathrm{M} t i}\right)$ For any $i(1 \leq i \leq 6)$, set of wffs $\Gamma$ and wff $A$, if $\Gamma \models_{\bar{M} t i}^{\leq} A$, then $\Gamma \vdash \stackrel{\leq}{\leq} A$.

Proof. Suppose $\Gamma \nvdash_{\mathrm{Lt} i}^{\leq} A$ for some $i(1 \leq i \leq 6)$, set of wffs $\Gamma$ and wff $A$. We prove $\Gamma \nvdash_{\mathrm{Mt} i}^{\leq} A$. If $\Gamma \nvdash_{\mathrm{Lt} i}^{\leq} A$, clearly $A \notin \mathrm{C} n \Gamma[\mathrm{Lt} i]$. Then, by the primeness lemma (Lemma 5.10), there is a prime Lti-theory $\mathcal{T}$ such that $\mathrm{C} n \Gamma[\mathrm{Lt} i] \subseteq \mathcal{T}$ and $A \notin \mathcal{T}$, whence it follows that $\mathcal{T}$ is, in addition, regular (since $\mathrm{Cn} \Gamma[\mathrm{Lt} i]$ is regular) and a-consistent. Suppose that $\mathcal{T}$ is inconsistent. Then, $\mathcal{T}$ is complete (by Proposition 5.9). Therefore, $\mathcal{T}$ generates an oLti-interpretation $I_{\mathcal{T}}$ such 
that $T \in I_{\mathcal{T}}(\mathcal{T})$ and $T \notin I_{\mathcal{T}}(A)$. Given that $\Gamma \subseteq \mathrm{C} n \Gamma[\operatorname{Lt} i] \subseteq \mathcal{T}, T \in I_{\mathcal{T}}(\Gamma)$. So, $\Gamma \nvdash_{\mathrm{Mt} i}^{\mathrm{o}} \leq$, by Definition 7.6.

On the other hand, suppose that $\mathcal{T}$ is consistent. Then, $\mathcal{T}$ generates a uLtiinterpretation $I_{\mathcal{T}}$ such that $T \in I_{\mathcal{T}}(\mathcal{T})$ and $T \notin I_{\mathcal{T}}(A)$. As $\mathcal{T}$ is consistent, there is no $B \in \mathcal{T}$ such that $F \in I_{\mathcal{T}}(B)$ : otherwise, $\neg B \in \mathcal{T}$ (by Lemma 6.8). So, $F \notin I_{\mathcal{T}}(\mathcal{T})$. Now, as $\Gamma \subseteq \mathrm{C} n \Gamma[\mathrm{Lt} i] \subseteq \mathcal{T}, T \in I_{\mathcal{T}}(\Gamma)$. Then, $\Gamma \nvdash_{\mathrm{Mt} i}^{\mathrm{u} \leq} A$ follows since $F \notin I_{\mathcal{T}}(\Gamma), T \notin I_{\mathcal{T}}(A)$ and $T \in I_{\mathcal{T}}(\Gamma)$ (cf. Definition 7.6). Therefore, if $\Gamma \nvdash_{\mathrm{Lt} i}^{\leq} A$, either $\Gamma \nvdash_{\mathrm{Mt} i}^{\mathrm{o}} A$ or $\Gamma \nvdash_{\mathrm{Mt} i}^{\mathrm{u} \leq} A$, whence $\Gamma \nvdash_{\mathrm{M} t i}^{\leq} A$ follows, by Proposition 7.8 , as was to be proved.

As a corollary, we have completeness w.r.t. $\models_{\mathrm{Mt} i}^{\mathrm{o} i}$ and $\models_{\mathrm{Mt} i}^{\mathrm{u} i}$.

Corollary 7.20 (Completeness of $\mathbf{L t} i \leq$ w.r.t. $\vDash_{\mathbf{L} t i}^{\mathbf{o} \leq}$ and $\vDash_{\mathbf{L t} i}^{\mathbf{u}} \leq$ ) For any $i(1$ $\leq i \leq 6$ ), set of wffs $\Gamma$ and wff $A$, (1) if $\Gamma \vDash_{L t i}^{o \leq} A$, then $\Gamma \vdash_{L t i}^{\leq} A$; and (2) if $\Gamma \vDash_{L t i}^{u \leq} A$, then $\Gamma \vdash \frac{\leq}{L t i} A$.

Proof. Immediate by Theorem 7.19 and Proposition 7.8.

\section{Concluding remarks}

We have the following facts concerning the logics determined by the 30 natural implicative expansions of MK3.

1. In [17], an o-determined truth-preserving consequence relation is used for axiomatizing the 24 natural implicative expansions of MK3 with two designated values (cf. Proposition 2.8 and Definition 2.9) in a unified and general way. That is, we have proved for any $i(1 \leq i \leq 24)$, set of wffs $\Gamma$ and wff $A, \Gamma \vdash_{\mathrm{Mti} i}^{2} A$ iff $\Gamma \vdash_{\mathrm{Lt} i}^{\mathrm{o} 2} A$ where $\left(\mathcal{L}, \vdash_{\mathrm{Lt} i}^{2}\right)$ is the logic determined by the matrix Mti and $\models_{\mathrm{Lt} i}^{\mathrm{o} i}$ is the aforementioned o-determined truth-preserving consequence relation.

2. In [19], a u-determined truth-preserving consequence relation is used for axiomatizing the logics determined by the 6 natural implicative expansions of MK3 with only one designated value (cf. Proposition 2.8 and Definition $2.9)$ in a general and unified way. That is, in the referred paper, it has been proved for any $i(1 \leq i \leq 6)$, set of wffs $\Gamma$ and wff $A, \Gamma \vdash_{\mathrm{Mt} i}^{2} A$ iff $\Gamma \models_{\mathrm{Lt} i}^{\mathrm{u} 2} A$ where $\left(\mathcal{L}, \vdash_{\mathrm{Lt} i}^{2}\right)$ is the logic determined by the matrix Mt $i$ and $\vDash_{\mathrm{Lt} i}^{\mathrm{u} 2}$ is the aforementioned u-determined truth-preserving consequence relation.

3. In the present paper, we have proved the following facts: (a) if understood as sets of theorems, or equivalently, as sets of valid formulas, the logics determined by the 6 natural implicative expansions of MK3 with only one designated value can be given equivalent $\mathrm{u}$-semantics and o-semantics; (b) for any $i(1 \leq i \leq 4), \mathrm{Lt} i \leq$ is sound and complete w.r.t. $\models_{\mathrm{M} t i}^{\leq}$. However, $\mathrm{Lt} 5 \leq$ (resp., Lt6 $\leq$ ) is only complete, not sound w.r.t. $\vDash_{\mathrm{M} t 5}^{\leq}\left(\right.$resp., $\left.\models_{\overline{\mathrm{M}} \mathrm{t} 6}^{\leq}\right)$. 
4. For any $i(1 \leq i \leq 4)$, $\mathrm{Lt} i \leq$ is a paraconsistent version of the logic $\left(\mathcal{L}, \vdash_{\mathrm{Lt} i}^{2}\right.$ ) treated in [19] (cf. point (2) above and Proposition 7.2). Thus, for example, Lt2 $\leq$ is the paraconsistent version of Łukasiewicz's 3-valued logic Ł3.

5. It is clear that the 24 logics investigated in [17] cannot be given a usemantics: the designated value 1 would have to be viewed as an underdetermined value. But an additional question is whether the logic Lt $i \leq$ is sound and complete w.r.t. $\models_{\mathrm{M} t i}^{\leq}(1 \leq i \leq 24)$. Concerning this question we note the two following facts.

(a) Consider the expansions of MK3 defined by the general table (designated values are starred):

TIII

\begin{tabular}{l|lll}
$\rightarrow$ & 0 & 1 & 2 \\
\hline 0 & 2 & $a_{1}$ & 2 \\
$*_{1}$ & 0 & $a_{2}$ & $a_{3}$ \\
$* 2$ & 0 & $a_{4}$ & 2
\end{tabular}

where $a_{i}(1 \leq i \leq 4) \in\{1,2\}$ (cf. Table I in Proposition 2.8). Let MtIII refer to any of the 16 expansions of MK3 definable from the tables in TIII (cf. Definition 2.9). LtIII is not sound w.r.t. $\vDash_{\mathrm{M} t I I I}^{\leq}$: for any MtIII-interpretation $I$ and wffs $A, B, I(A \rightarrow B)=2$ or $I(A \rightarrow B)=1$ whenever $I(A)=2$ and $I(B)=1$.

(b) Consider the expansions of MK3 defined by the general table (designated values are starred):

TIV

\begin{tabular}{l|lll}
$\rightarrow$ & 0 & 1 & 2 \\
\hline 0 & 2 & $a_{1}$ & 2 \\
$*_{1}$ & 0 & $a_{2}$ & $a_{3}$ \\
$* 2$ & 0 & 0 & 2
\end{tabular}

where $a_{i}(1 \leq i \leq 3) \in\{1,2\}$ (cf. Table I in Proposition 2.8). Let MtIV refer to any of the 8 expansions of MK3 definable from the tables in TIV (cf. Definition 2.9). LtIV is sound and complete w.r.t. $\kappa_{\mathrm{M} t I V}^{\leq}$: in fact, it would not be difficult to prove that $\mathrm{Lt}_{\mathrm{tIV}}^{\leq}$and $\mathrm{Lt}_{\mathrm{tIV}}^{2}$ are equivalent logics (cf. Definition 2.2).

6. The referees of the IGPL have called our attention to some work related to the research recorded in our paper. In particular, [14] and [15] study the logics described in Definition 2.9 in a natural deduction setting, while [11] investigates truth-functional extensions of Priest's LP in the same context. On the other hand, in [13], BD-semantics is defined for truth-functional expansions of Anderson and Belnap's First Degree Entailment Logic, FDE, Priest's LP and Kleene's K3. Then, in [21], natural deduction systems for the first order Belnap-Dunn logics are systematically explored by essentially using Baaz' delta operator. Finally, [22] proposes an extension of LP with Rescher's implication. 
Funding. This work was supported by the Spanish Ministry of Economy, Industry and Competitiveness [FFI2014-53919-P, FFI2017-82878-P]. - We sincerely thank three referees of the IGPL for their comments and suggestions on a previous draft of this paper.

\section{References}

[1] Anderson, A. R., Belnap, N. D. Jr. (1975). Entailment. The Logic of Relevance and Necessity, vol I. Princeton, NJ: Princeton University Press.

[2] Arieli, O., Avron, A. (1996). Reasoning with logical bilattices. Journal of Logic, Language and Information, 5(1), 25-63.

[3] Arieli, O., Avron, A. (1998). The value of the four values. Artificial Intelligence, 102, 97-141.

[4] Avron, A. (1991). Natural 3-Valued Logics-Characterization and Proof Theory. Journal of Symbolic Logic, 56(1), 276-294.

[5] Belnap, N. D. Jr. (1977). How a computer should think. In G. Ryle (Ed.), Contemporary Aspects of Philosophy (pp. 30-55). Oriel Press Ltd., Stocksfield.

[6] Belnap, N. D. J.r (1977). A useful four-valued logic. In G. Epstein \& J. M. Dunn (Eds.), Modern Uses of Multiple-Valued Logic (pp. 8-37). D. Reidel Publishing Co., Dordrecht.

[7] Dunn, J. M. (1976). Intuitive semantics for first-degree entailments and "coupled trees." Philosophical Studies, 29, 149-168.

[8] Dunn, J. M. (2000). Partiality and its Dual. Studia Logica, 65, 5-40. https://doi.org/10.1023/A:1026740726955.

[9] González, C. (2012). MaTest. Retrieved from http://ceguel.es/matest (last accessed 09/12/2018).

[10] Kleene, S. C. (1952). Introduction to Metamathematics. North Holland. Reprinted Ishi Press, 2009.

[11] Kooi, B., Tamminga, A. (2012). Completeness via correspondence for extensions of the logic of paradox. The Review of Symbolic Logic, 5(4), 720730. https://doi.org/10.1017/S1755020312000196

[12] Kracht, M. (1998). On Extensions of Intermediate Logics by Strong Negation. Journal of Philosophical Logic, 27(1), 49-73. https://doi.org/10.1023/A:1004222213212.

[13] Omori, H., Sano, K. (2015). Generalizing Functional Completeness in Belnap-Dunn Logic. Studia Logica, 103(5), 883-917. https://doi.org/10.1007/s11225-014-9597-5. 
[14] Petrukhin, Y. (2017). Natural Deduction for Three-Valued Regular Logics. Logic and Logical Philosophy, 26(2), 197-206. https://doi.org/10.12775/LLP.2016.025.

[15] Petrukhin, Y., Shangin, V. (2018). Natural three-valued logics characterized by natural deduction. Logique et Analyse, 61(244).

[16] Robles, G., Méndez, J. M. (2014). A paraconsistent 3-valued logic related to Gödel logic G3. Logic Journal of the IGPL, 22 (4), 515-538. https://doi.org/10.1093/jigpal/jzt046.

[17] Robles, G., Méndez, J. M. (2019). Belnap-Dunn semantics for natural implicative expansions of Kleene's strong three-valued matrix with two designated values. Journal of Applied Non-Classical Logics, 29(1), 37-63. https://doi.org/10.1080/11663081.2018.1534487.

[18] Robles, G., Salto, F., Méndez, J. M. (2014). Dual Equivalent Two-valued Under-determined and Over-determined Interpretations for Lukasiewicz's 3-valued Logic Ł3. Journal of Philosophical Logic, 43 (2/3), 303-332. https://doi.org/10.1007/s10992-012-9264-0.

[19] Robles, G., Salto, F., Méndez, J. M. (Forthcoming). Belnap-Dunn semantics for natural implicative expansions of Kleene's strong three-valued matrix II. Only one designated value. Journal of Applied Non-Classical Logics.

[20] Routley, R. Meyer, R. K., Plumwood, V., Brady R. T. (1982). Relevant Logics and their Rivals, vol. 1. Atascadero, CA: Ridgeview Publishing Co.

[21] Sano, K., Omori, H. (2014). An expansion of first-order Belnap-Dunn logic. Logic Journal of the IGPL, 22(3), 458-481. https://doi.org/10.1093/jigpal/jzt044.

[22] Thomas, N. (2013). LP=>: Extending LP with a strong conditional operator. Cornell University ArXiv.Org - Eprint ArXiv:1304.6467. Available at https://arxiv.org/abs/1304.6467v1.

[23] Tomova, N. (2010). Implicative extensions of regular Kleene logics. Logical Investigations, 16. M.-SPb: C.G.I. 233-258.

[24] Tomova, N. (2012). A Lattice of implicative extensions of regular Kleene's logics. Reports on Mathematical Logic, 47, 173-182. https://doi.org/ 10.4467/20842589RM.12.008.0689.

[25] Tomova N. (2013). Natural three-valued logics and classical logic. Logical Investigations, 19 (Special Issue). M. Spb.: C.G.I., 344-352.

[26] Wójcicki, R. (1984). Lectures on Propositional Calculi. Pub. House of the Polish Academy of Sciences. 